\title{
UVB mutagenesis differs in NRAS- and BRAF-mutant mouse models of melanoma
}

\author{
Robert L. Bowman ${ }^{1 *}$, Rebecca C. Hennessey ${ }^{2 *}$, David A. Tallman ${ }^{2,3}$, Tirzah J. Weiss ${ }^{2,3}$, \\ Emma R. Crawford ${ }^{3}$, Brandon M. Murphy ${ }^{2}$, Amy Webb ${ }^{4}$, Souhui Zhang ${ }^{2}$, Krista M. D. La \\ Perle $^{5}$, Craig J. Burd ${ }^{3}$, Ross L. Levine ${ }^{1}$, A. Hunter Shain ${ }^{6}$ and Christin E. Burd ${ }^{2,3 \dagger}$ \\ ${ }^{1}$ Human Oncology and Pathogenesis Program, Memorial Sloan Kettering Cancer Center, New \\ York, NY 10065 \\ ${ }^{2}$ Departments of Cancer Biology and Genetics, ${ }^{3}$ Molecular Genetics, ${ }^{4}$ Biomedical Informatics, \\ and ${ }^{5}$ Veterinary Biosciences, The Ohio State University, Columbus, Ohio, 43210 \\ ${ }^{6}$ Department of Dermatology, Helen Diller Family Comprehensive Cancer Center, University of \\ California, San Francisco (UCSF), San Francisco, CA 94115 \\ *Authors contributed equally \\ ${ }^{\dagger}$ Address correspondence to: \\ Christin E. Burd, Ph.D. \\ The Ohio State University \\ Biomedical Research Tower, Rm 918 \\ 460 W. $12^{\text {th }}$ Avenue \\ Columbus, Ohio 43210, USA \\ Phone: (614)688-7569 \\ Fax: (614)292-6356 \\ Email: burd.25@osu.edu
}

RUNNING TITLE

Enhanced UVB carcinogenesis in BRAF-mutant murine melanoma

KEY WORDS

Mutational signature, UVA, UVB, melanoma, carcinogenesis 


\section{ABSTRACT}

$B R A F$-mutant melanomas are more likely than NRAS-mutant melanomas to arise in anatomical locations protected from chronic sun damage. We hypothesized that this discrepancy in tumor location is a consequence of the differential sensitivity of BRAF and NRAS-mutant melanocytes to ultraviolet light (UV)-mediated carcinogenesis. We tested this hypothesis by comparing the mutagenic consequences of a single, narrow band ultraviolet-A (UVA; 320-400nm) or ultravioletB (UVB; 280-320nm) exposure in mouse models predisposed to Braf- or Nras-mutant melanoma. Exposures approximated the amount of UVA or UVB energy contained in $\sim 40$ minutes of summer sunlight. Tumor onset was accelerated in all UVB-, but only half of UVA- irradiated mice as compared to unirradiated controls. Melanomas from both mouse models, harbored recurrent

11 mutations affecting the RING domain of MAP3K1 and Actin-binding domain of Filamin A 12 irrespective of UV status. Melanomas from UVB-irradiated, Braf-mutant mice averaged twice as many SNVs (1,025 vs. 435$)$ and five times as many dipyrimidine variants (33.3 vs. 5.7$)$ than

14 tumors from similarly irradiated Nras-mutant mice. We identified a mutational signature enriched

15 in UVB-accelerated tumors which mirrored COSMIC signatures associated with human skin 16 cancer. Notably, this signature was enriched to a greater extent in Braf- than Nras-mutant murine

17 melanomas. These data suggest that oncogenic BRAF may enhance UVB carcinogenesis to promote melanoma formation at anatomic sites with low or intermittent sun exposure. 


\section{INTRODUCTION}

The most common genetic subtypes of human melanoma, NRAS- and BRAF-mutant, are enriched in different anatomical locations. NRAS-mutant melanomas preferentially localize to chronically sun-damaged (CSD) skin on the head and neck, whereas BRAF-mutant melanomas are more common in areas of intermittent sun exposure (Zhang et al. 2016). Despite the association of NRAS-mutant tumors with CSD skin, it is reported that UV signature lesions (C>T and $\mathrm{CC}>\mathrm{TT})$ are prevalent in a similar proportion of NRAS- and BRAF-mutant melanomas (Cancer Genome Atlas 2015). These observations led us to speculate that BRAF-mutant nevi could be more sensitive to UV mutagenesis and subsequent melanocyte transformation. However, it is difficult to control for differences in lifetime sun exposure among biopsies of human melanomas or nevi.

Genetically engineered mouse models (GEMMs) provide a controlled genetic background in which the genomic and phenotypic effects of UV exposures can be studied. GEMMs encoding a melanocyte-specific Nras- or Braf-mutation mimic the presense of these mutations in human benign nevi (Roh et al. 2015). Moreover, neonatal or chronic UV treatment accelerates the formation and progression of melanoma in a variety of melanoma GEMMs, consistent with human disease etiology (Mukhopadhyay et al. 2016; Chagani et al. 2017; Hennessey et al. 2017; PerezGuijarro et al. 2017; Trucco et al. 2019). Genomic analyses of tumors from UV-treated Nras or genomic effects of narrow band UVA (360-390 nm) and UVB (280-320 nm) exposures in NrasBraf-mutant GEMMs have been reported (Viros et al. 2014; Mukhopadhyay et al. 2016; Trucco et al. 2019). However, no study has directly compared the mutational profiles of Nras- and Brafdriven mouse melanomas exposed to the same UV dosing scheme. Therefore, a complete understanding of how different oncogenic drivers cooperate with environmental mutagens to promote transformation is lacking.

Here, we used a single-dose UV irradiation scheme to characterize the phenotypic and and Braf-mutant mouse models of melanoma. We exposed these animals to a single dose of UVA 
45 or UVB, approximating the amount of energy from each band of the UV spectrum in 40 minutes

46 of intense sunlight. Then, we monitored the mice for melanoma development. Tumors from these

47 animals were sequenced to gain insight into the mutational consequences of each UV source in

$48 \quad$ Nras- and Braf-mutant melanocytes. 


\section{RESULTS}

50

51

52

53

54

\section{UV exposure alters NRAS- and BRAF-mutant melanomagenesis}

We generated melanocyte-specific, Tyr::CreER(T2)-driven, Nras (TN) and Braf (TB) mice to model the major genetic subtypes of human melanoma (Hodis et al. 2012) (Figure 1A-B). TN mice are homozygous for the LSL-Nras ${ }^{Q 61 R}$ allele (Burd et al. 2014; Hennessey et al. 2017), whereas TB animals carry a heterozygous, conditional Braf ${ }^{\vee 637 E}$ allele (Braf ${ }^{C A}$; (Dankort et al. 2007). Notably, the Braf ${ }^{\vee 637 E}$ allele is the murine equivalent of human Braf ${ }^{\vee 600 E}$ (Rad et al. 2013).

Oncogene expression is driven by the endogenous gene promoter in both models, and is activated by a melanocyte-specific, tamoxifen-inducible Cre recombinase (Tyr::CreER(T2); (Bosenberg et al. 2006)). Therefore, the expression of oncogenic Nras or Braf in these mice mimics the presence of NRAS and BRAF mutations in most benign human nevi (Roh et al. 2015). Mice carrying only the Braf ${ }^{C A}$ allele rarely develop melanoma (Dankort et al. 2009). For this reason, $p 16^{\text {INK4a }}$ conditional knockout alleles ( $p 16^{L}$; (Monahan et al. 2010)) were included in both the $T N$ and TB models. While p16 $16^{\text {INK4a }}$ loss-of-function is an early event observed in $>60 \%$ of human melanomas, germline mutations affecting $p 16^{I N K 4 a}$ are insufficient to drive the disease in mice or humans (Bishop et al. 2000; Shain et al. 2015b; Hennessey et al. 2017).

$T N$ and $T B$ mice were topically treated with 4-hydroxytamoxifen (4OHT) on postnatal days one and two to induce Cre activity and stimulate recombination of the conditional p16 INK4a knockout and $L S L-N r^{61 R}{ }^{61 R}$ or Braf ${ }^{C A}$ alleles. On postnatal day three, the mice were exposed to a single dose of ambient light ("No UV" or $0 \mathrm{~kJ} / \mathrm{m}^{2}$ ), narrow band UVA or narrow band UVB irradiation. The amount of UVB delivered approximated that which is contained in 40 minutes of summer sunlight $\left(4.5 \mathrm{~kJ} / \mathrm{m}^{2}\right)$, whereas the amount of UVA employed models a short, indoor tanning session (70 or $150 \mathrm{~kJ} / \mathrm{m}^{2}$; see: Methods). These dosing schemes approximate sun exposures of a similar duration, as the UVB to UVA ratio in sunlight is $\sim 1: 20$, but varies based on season, cloud cover and latitude (Cadet and Douki 2018). Neither dose of UVA or UVB caused erythema or blistering. 
bioRxiv preprint doi: https://doi.org/10.1101/778449; this version posted December 27,2020 . The copyright holder for this preprint (which was
not certified by peer review) is the author/funder. All rights reserved. No reuse allowed withoutpermission A not certified by peer review) is the author/funder. All rights reserved. No reuse allowed without permis
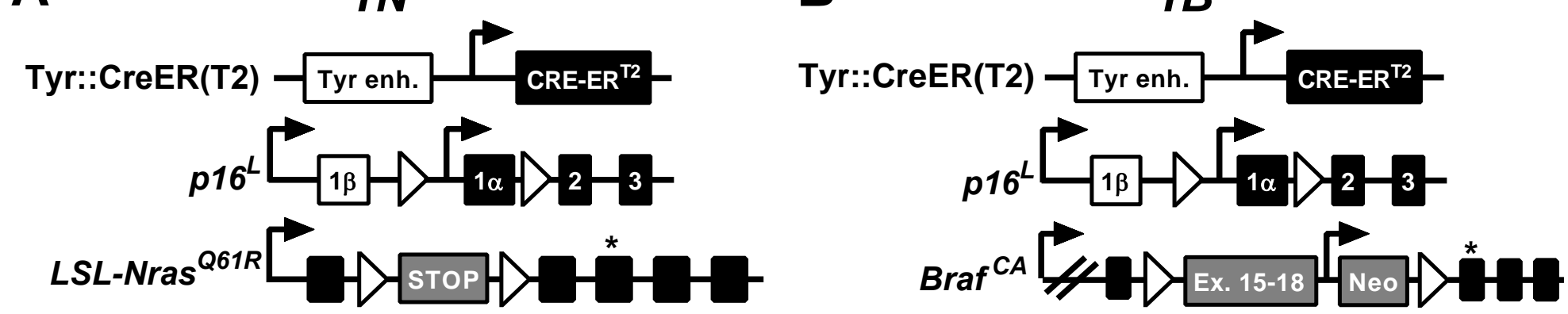

C

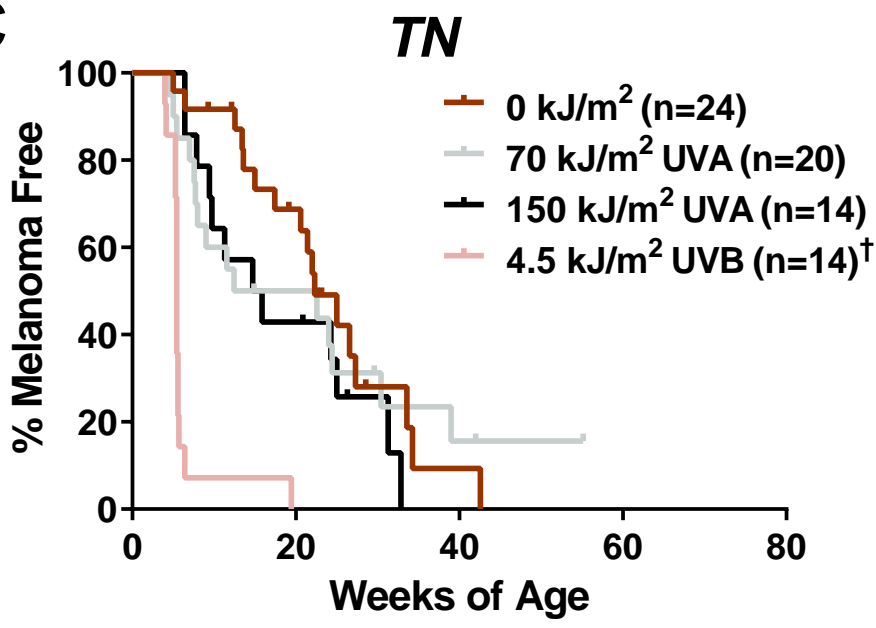

E

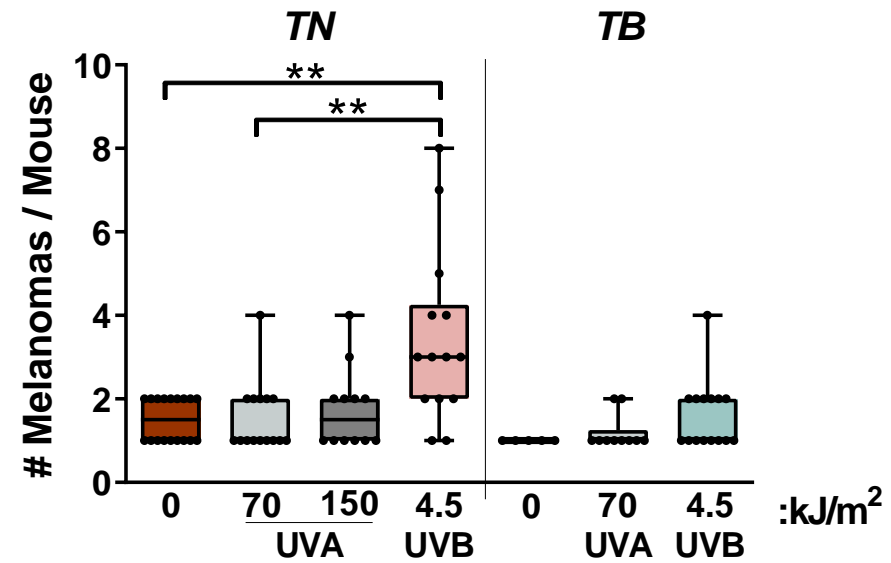

D
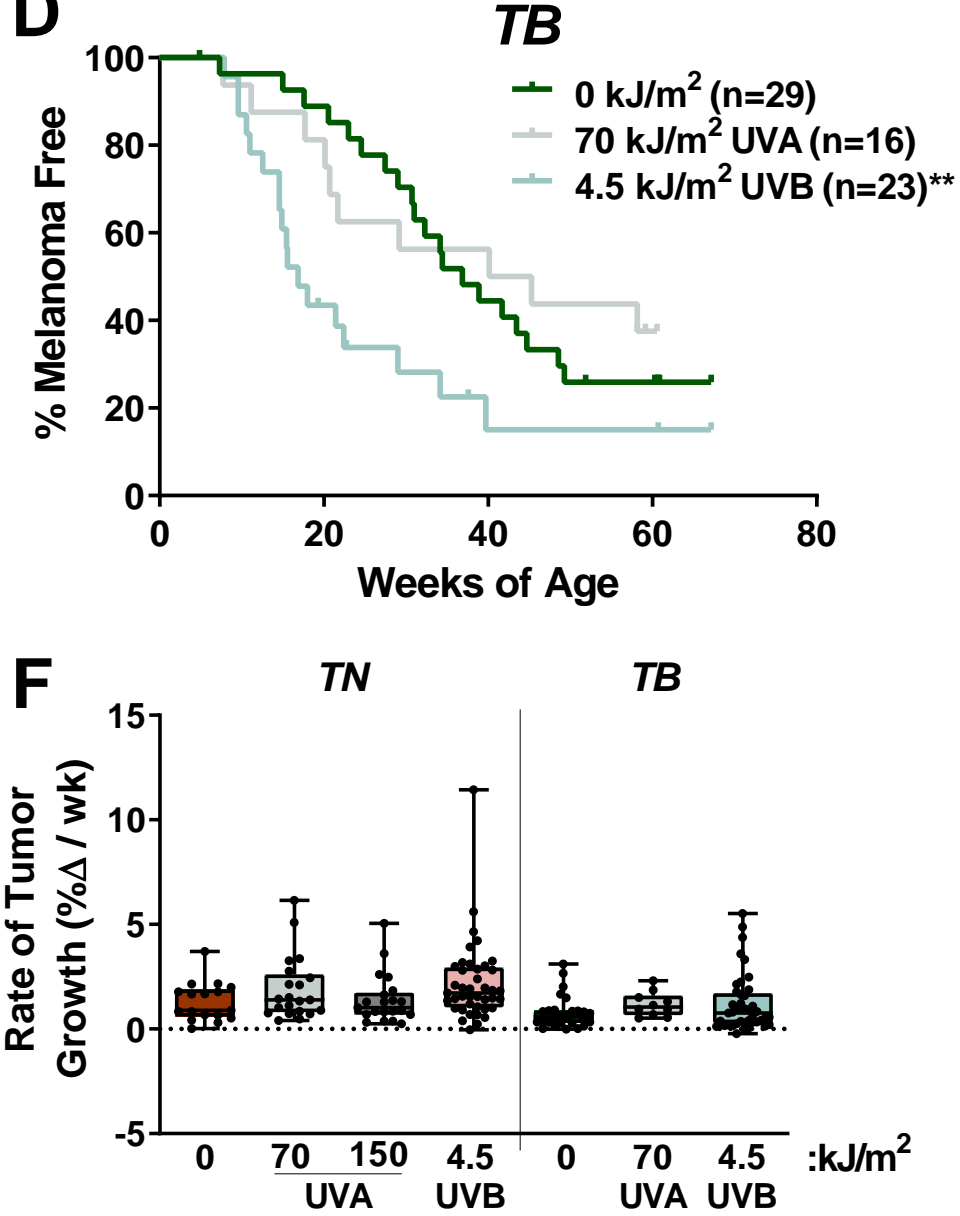

Figure 1. Neonatal UV exposure alters melanoma onset in TN and TB mice. A, TN mice are homozygous for a melanocyte-specific, tamoxifen-inducible Cre transgene (Tyr::CreER(T2)), a conditional $p 16^{I N K 4 a}$ knockout allele $\left(p 16^{L}\right)$, and a conditional $\operatorname{Nras}^{Q 61 R}$ knock-in allele $\left(L S L-N r a s^{Q 61 R}\right)$. Open triangles represent LoxP sites. A star indicates the location of the Nras ${ }^{Q 61 R}$ mutation. B, TB mice carry a single, Braf ${ }^{V 637 E}$ conditional allele (LSL$B$ Braf ${ }^{C A}$ ) and are homozygous for Tyr::CreER(T2) and $p 16^{L}$. Note that BRAF ${ }^{V 37 E}$ is the murine equivalent of human BRAF ${ }^{V 600 E}$. Open triangles represent LoxP sites and the location of the V637E mutation is indicated by a star. C \& D, Kaplan-Meier curves depicting the melanoma-free survival of $T N(C)$ and $T B(D)$ mice treated on postnatal day three with a single dose of ambient light $\left(0 \mathrm{~kJ} / \mathrm{m}^{2}\right)$, UVA or UVB. $\dagger=p<0.0001,{ }^{* *}=p<0.01$ comparing control $\left(0 \mathrm{~kJ} / \mathrm{m}^{2}\right)$ and UV-irradiated animals of the same genotype (Gehan-Breslow-Wilcoxon). E, Total tumor burden of control and UV-irradiated $T N$ and $T B$ mice at euthanasia. Each circle represents a single mouse. Boxes represent the mean and interquartile range for each group. Whiskers span from the minimum to the maximum value. ${ }^{* *}=p<0.01$ comparing control $\left(0 \mathrm{~kJ} / \mathrm{m}^{2}\right)$ animals of the same genotype (two-tailed, unpaired t-tests with Welch's correction). F, Average tumor growth rates for UV- and mock-irradiated TN and TB mice. Each circle represents a single tumor $\left(T N: 0 \mathrm{~kJ} / \mathrm{m}^{2} \mathrm{n}=17 ; 70 \mathrm{~kJ} / \mathrm{m}^{2}\right.$ UVA $\mathrm{n}=21 ; 150 \mathrm{~kJ} / \mathrm{m}^{2} \mathrm{UVA} \mathrm{n}=21$; $4.5 \mathrm{~kJ} / \mathrm{m}^{2}$ UVB $\mathrm{n}=41 ;$ TB: $0 \mathrm{~kJ} / \mathrm{m}^{2} \mathrm{n}=27 ; 70 \mathrm{~kJ} / \mathrm{m}^{2}$ UVA $\left.\mathrm{n}=10 ; 4.5 \mathrm{~kJ} / \mathrm{m}^{2}, \mathrm{n}=41\right)$. Data are presented as described in ' $E$ '. 
The onset of spontaneous melanoma was compared among mice exposed to No UV, UVA or UVB irradiation. Exposure to a single dose of $4.5 \mathrm{~kJ} / \mathrm{m}^{2}$ UVB dramatically accelerated melanoma onset and decreased overall survival in both the TN and TB models (Figures 1C-D, Suppl. Figure S1). Exposure to $70 \mathrm{~kJ} / \mathrm{m}^{2}$ UVA also enhanced melanoma formation, but only half of the mice developed tumors earlier than the median onset in unirradiated animals (Figure 1C-D). Doubling this dose of UVA in the TN model did not further facilitate melanoma formation, suggesting that $70 \mathrm{~kJ} / \mathrm{m}^{2}$ UVA was sufficient to elicit the maximal response achievable with a single exposure (Figure 1C). Together, these results reveal the potent ability of narrow band UVB to promote melanoma formation in $T N$ and $T B$ mice. Furthermore, our findings suggest that UVA could facilitate melanoma onset in some settings, albeit to a much lesser extent than UVB.

We next examined the incidence and growth phenotypes of tumors arising in each of our experimental cohorts. Tumor incidence (\# melanomas/mouse) increased in UVB-exposed TN mice, but was not significantly altered in TN animals treated with UVA or TB mice exposed to any form of UV (Figure 1E). Tumor distribution and incidence were also similar between male and female $T N$ and TB mice regardless of exposure, with $\sim 59 \%$ of tumors arising on the trunk, $\sim 13 \%$ on the head and $\sim 16 \%$ on the ears or tail (data not shown). Once established, TN and TB tumors grew at the same rate regardless of prior exposure (Figure 1F). Therefore, early tumor onset, rather than more rapid melanoma growth, is responsible for the reduction in overall survival observed in UVB-exposed TN and TB mice.

We postulated that melanomas arising in UVA- or UVB-exposed mice would exhibit distinct histopathological features. Therefore, we examined hematoxylin and eosin stained tumor sections representative of the rate of onset and body site distribution of melanomas from each cohort. Tumors from both models contained variable percentages of myxoid and spindle cells with comparable degrees of invasion, mitosis and granulocyte infiltration regardless of treatment (Suppl. Figure S2A-C, data not shown). A paucity of pilosebaceous units and hyperplasia of the overlying epidermis was also observed in UVA, UVB and unexposed mice of both genotypes 
101

102

103

104

105

106

107

108

109

110

111

112

113

114

115

116

117

118

119

120

121

122

123

124

125

126

(Suppl. Figure S2A\&F). Most tumors from the UVB-treated TN cohort contained neoplastic cells with plasmacytoid features that were not prevalent in TN melanomas from the UVA and No UV cohorts ( 6 of 7 vs. 2 of 6 and 0 of 5 tumors, respectively; Suppl. Figure S2D). Fibroblastic features were seen in TN melanomas from UVA-treated animals (3 of 6), but were not overtly apparent in tumors from other $T N$ mice (Suppl. Figure S2E). Unlike the $T N$ model, tumor samples from TB mice contained areas of pigmentation, typically characterized by multiple clusters of melanophages distributed at the dermal-hypodermal interface with or without associated neoplastic cells and occasionally within the tumors (Suppl. Figure S2F). These results show that although the histopathological features of cutaneous murine and human melanomas differ, a single UVA or UVB exposure can promote the formation of cutaneous, murine tumors with distinct morphologic features.

\section{Identification of clustered FIna and Map3k1 mutations in TN and TB melanomas}

Prior GEMM studies revealed an enrichment of Trp53 mutations in melanomas accelerated by full-spectrum- (UVA + UVB) or UVB-irradiation (Viros et al. 2014; Mukhopadhyay et al. 2016; Trucco et al. 2019). However, Trp53 mutations occur late in human melanoma pathogenesis (Shain et al. 2018). We sought to identify variants associated with earlier stages of melanoma progression, and performed WES using an ensemble calling approach to identify variants in $T N$ and TB melanomas (see Methods). Pooled normals from each inbred mouse model served as germline controls and polymorphisms observed in dbSNP were excluded (Kitts et al. 2013).

Unlike previous reports, Trp53 mutations were extremely rare in melanomas from our models (1 of 36 tumors, Suppl. Table 1). Instead, clustered, recurrent Flna and Map3k1 alterations were observed in tumors from multiple $T N$ and $T B$ litters (Figure 2A-C). Thirteen of the 15 identified FIna mutations (87\%) localized to the tenth Ig-like repeat of Filamin A (Figure 2B). Alterations in this domain are reported to alter Filamin A binding to F-ACTIN and may also affect protein translation and stability (Nakamura et al. 2007; Page et al. 2011; Suphamungmee et al. 2012). 


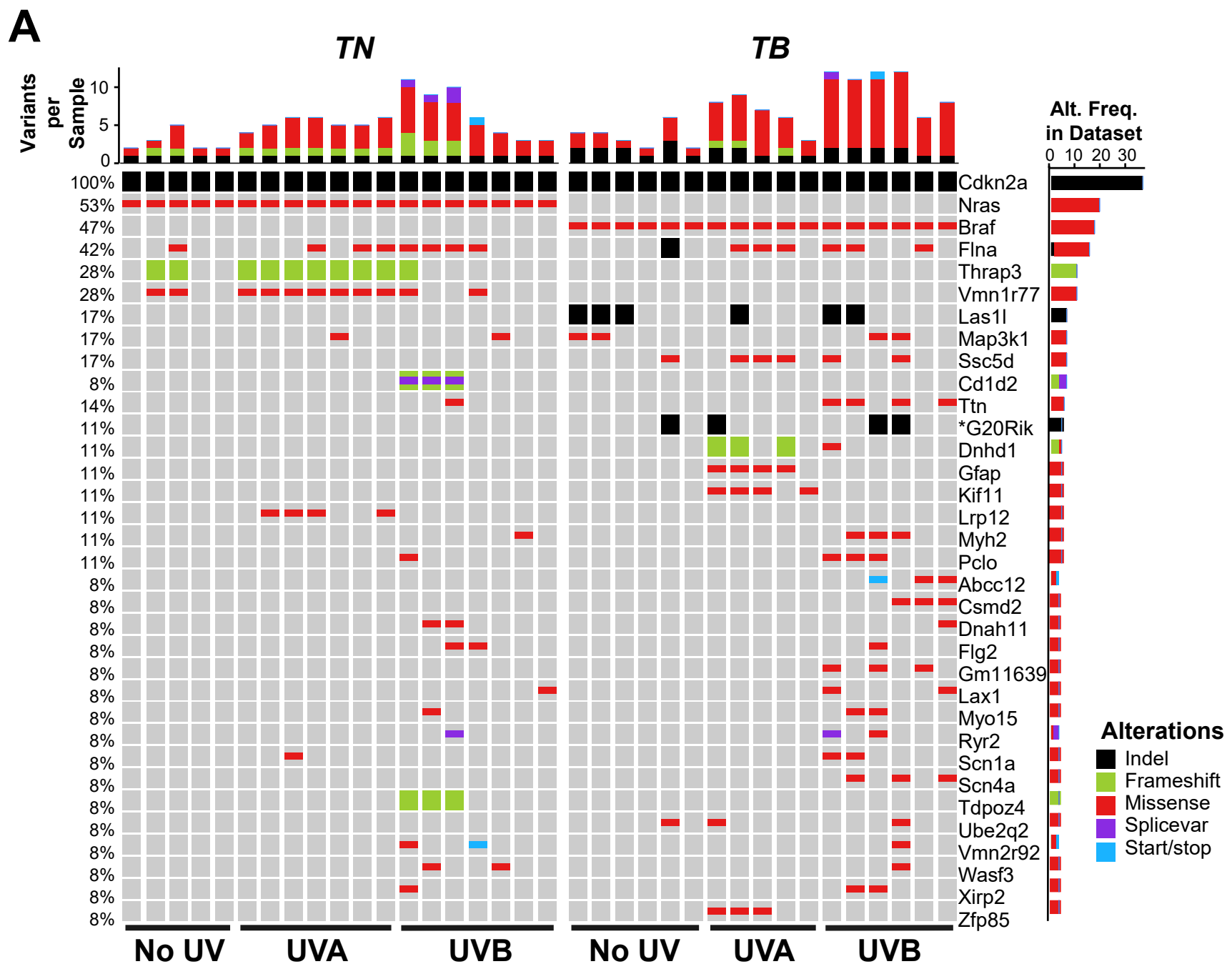

B

Filamin A

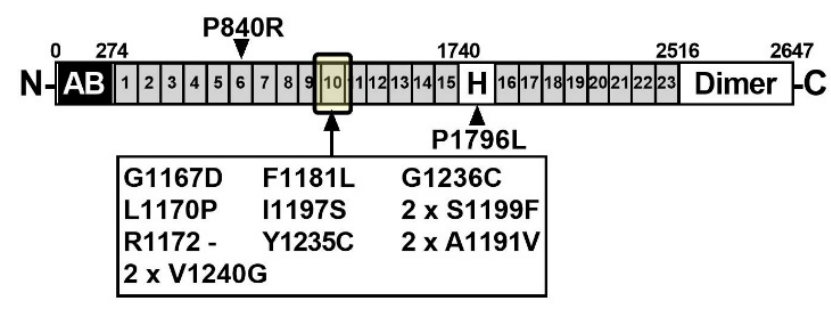

C

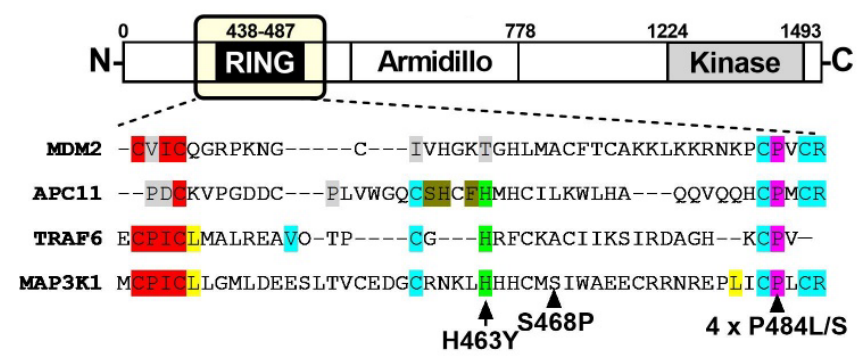

Figure 2. Recurrent genetic alterations in murine models of UV-associated melanomagenesis. A, Oncoprint depicting genes mutated in $\geq 3 T N$ and $T B$ tumors treated with no UV, UVA or UVB. Color is used to indicate each mutation type: SNV, indel, frameshift, splice variant or nonsense/stop. Total mutation burden is shown at the top of each sample column. The frequency at which each gene is altered in the dataset is indicated to the right of each row. B, Schematic depicting the protein domains of Filamin A. A recurrent cluster of mutations was identified in the tenth Ig-like repeat domain as indicated by the arrow. C, Schematic depicting the protein domains of MAP3K1, where recurrent mutations were identified in the RING domain (arrows). Bottom panel depicts a multiple sequence alignment of related E3 ubiquitin ligases, highlighting the conservation of mutated residues. 
Two $T N$, and four TB melanomas, contained mutations affecting conserved residues of the MAP3K1 RING domain (Figure 2C). These findings are consistent with prior publications implicating MAP3K1 and Filamin A in melanoma progression (Ni et al. 2013; Savoy and Ghosh 2013; Mann et al. 2015; Trucco et al. 2020).

\section{UVB increases the SNV burden of TN and TB melanomas}

In contrast to human melanomas, tumors from genetically engineered mouse models are frequently characterized by a high burden of genomic copy number alterations (CNAs) and few single nucleotide variants (SNVs) (Hodis et al. 2012; Krauthammer et al. 2012; Zhang et al. 2016; Wang et al. 2017; Zloza et al. 2017). Thus, we sought to determine whether a single UVA or UVB exposure caused significant alterations to the genomic landscape of $T N$ or TB tumors. Fewer CNAs were seen in all TN tumors exposed to UVB and in 5 of $7 T N$ tumors exposed to UVA as compared to melanomas from unirradiated controls (Figure 3A-B). Conversely, only 3 of 6 melanomas from our UVB-irradiated $T B$ mice had a lower CNA burden than tumors from unirradiated controls (Figure 3A-B). The most common CNA observed in TB tumors was a gain in chromosome 6, the chromosome in which Braf resides (Figure 3A). Consistent with this observation, 5 of 12 TB melanomas showed increased BRAF protein expression as compared to normal, murine skin (Figure S3). Recurring copy number gains in chromosomes 1 and 10 were also observed in tumors from the TB-UVA, TN-No UV and TN-UVA groups (Figure 3A).

The average burden of SNVs increased in both $T N$ and $T B$ melanomas as a result of prior UVB exposure, whereas the SNV burden of UVA-irradiated TN and TB tumors was slightly higher, but not statistically different, than that observed in tumors from the No UV groups (Figure $3 \mathrm{C}$; 0.67 vs. 0.93 and 1.44 vs. $0.65 \mathrm{SNVs} / \mathrm{Mb}$ on average, respectively). The frequency of insertions and deletions (indels) did not differ in tumors from irradiated and unirradiated TB mice, but increased in $T N$-UVB melanomas as compared to unirradiated controls (Figure 3D). Melanomas from UVB-irradiated TN and TB mice were also enriched for dypyrimidine substitutions, consistent 
A

\section{TN}

TB
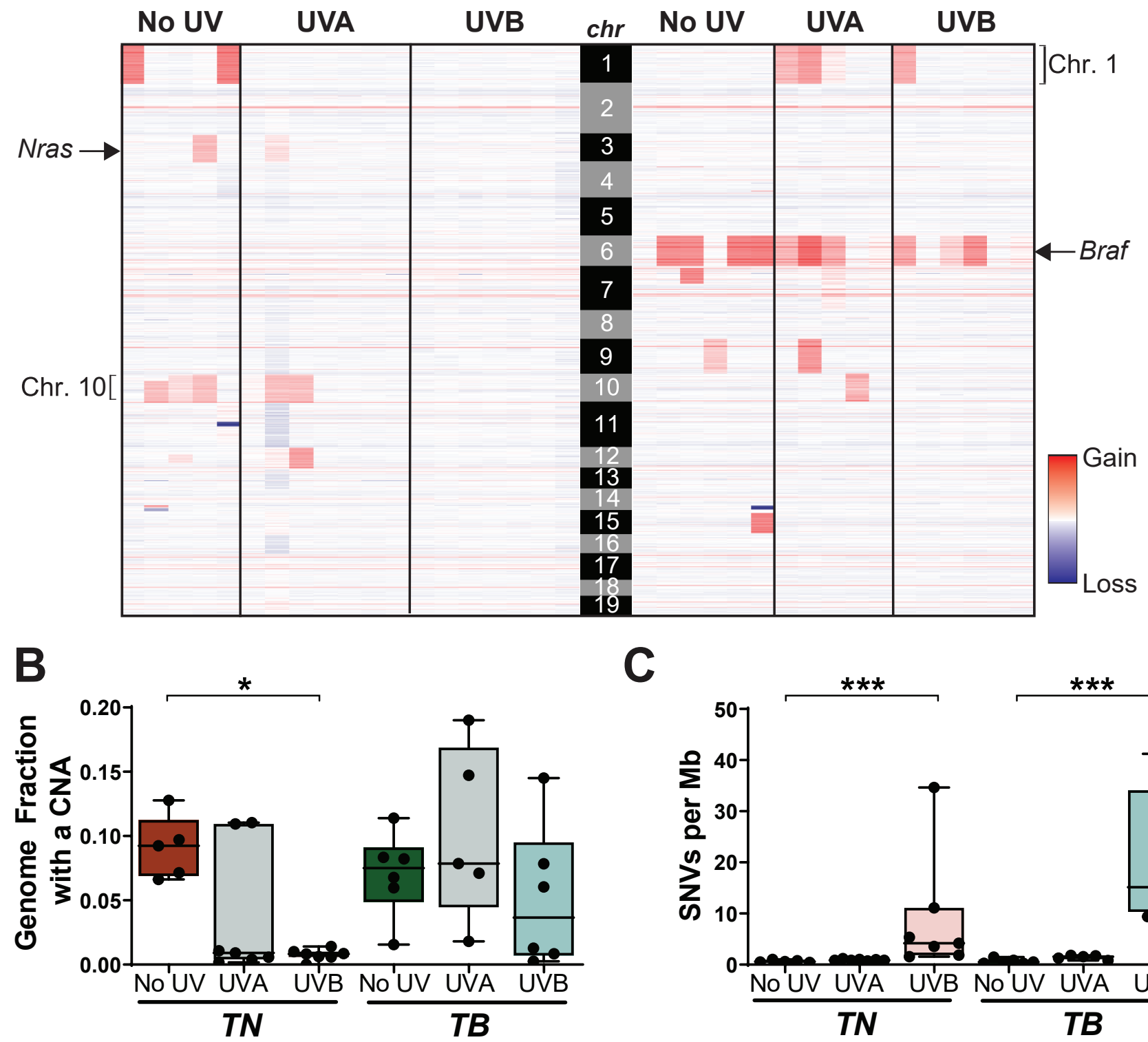

C
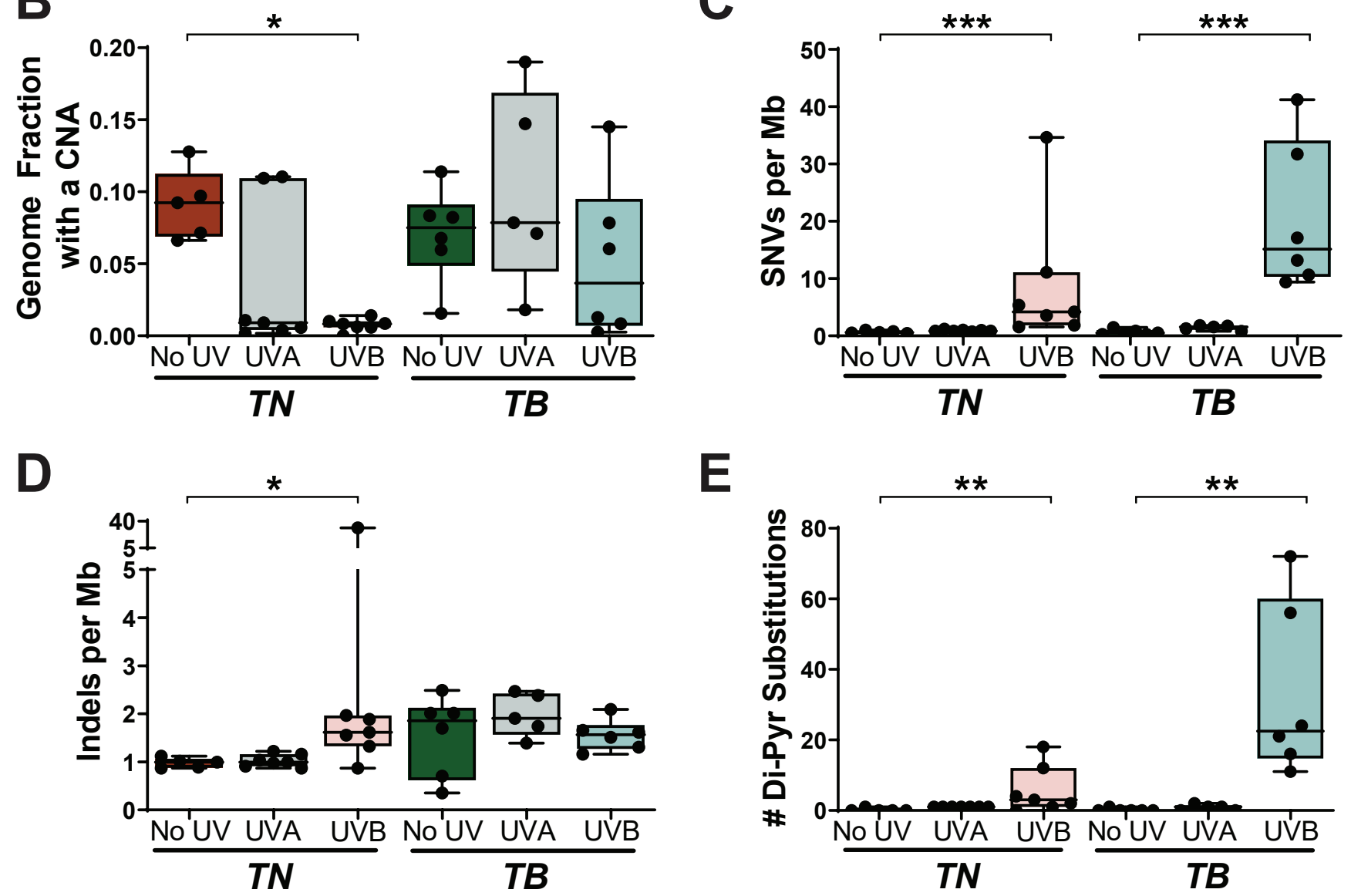

Figure 3. UV alters the genomic landscape of $T N$ and $T B$ tumors. A, Heatmap showing areas of genomic gain or loss within each sequenced tumor. Columns correspond to individual tumors and rows correspond to genomic bins. B, Fraction of each sequenced melanoma genome exhibiting a copy number alteration (CNA), graphed as a box plot with whiskers indicating the $5^{\text {th }}$ and $95^{\text {th }}$ percentiles. Dots represent individual tumors. p-values determined using a Kruskal-Wallis test with Dunn's correction. C-D, Single nucleotide variants (SNVs) (C) and indels (D) per megabase (Mb) of captured genome, plotted and analyzed as in 'B'. E, Number of dipyrimidine substitutions per tumor, plotted and analyzed as in 'B'. For B-E: ${ }^{*}=p<0.05,{ }^{* *}=p<0.01,{ }^{* * *}=p<0.001$. 
153

154

155

156

157

158

159

160

161

162

163

164

165

166

167

168

169

170

171

172

173

174

175

176

177

178

with the ability of UVB to promote cyclobutane pyrimidine dimer (CPD) formation (Figure 3E; (Cadet and Wagner 2013)).

\section{UVB drives genotype-dependent mutagenesis on the non-transcribed DNA strand}

UVA is the most prevalent form of UV in terrestrial sunlight; however, it is poorly absorbed by DNA (Setlow 1974; Sutherland and Griffin 1981; Pfeifer et al. 2005; Khan et al. 2018). By contrast, UVB can directly damage DNA and is the major form of UV responsible for skin erythema and many skin cancers (Setlow 1974). Both bands of the UV spectrum generate reactive species that promote the formation of a wide variety of modified nucleotides (Cadet and Wagner 2013). To examine whether distinct mutation types arise after UVA or UVB irradiation, we quantified the burden of each SNV type $(\mathrm{C}>\mathrm{A}, \mathrm{C}>\mathrm{G}, \mathrm{C}>\mathrm{T}, \mathrm{T}>\mathrm{A}, \mathrm{T}>\mathrm{C}$ or $\mathrm{T}>\mathrm{G})$ in our sequenced $T N$ and $T B$ melanomas (Suppl. Table 2A). We also examined the prevalence of C>T transitions at CpG sites because methylated cytosines are reported to form cyclobutane pyrimidine dimers (CPDs) with higher efficiency than non-methylated cytosines (Tommasi et al. 1997).

We compared both the absolute number and relative frequency of each mutation type between tumor types from each genotype and UV irradiation status (Suppl. Table 2B-C). As anticipated, melanomas from UVB-treated TB mice had a greater number of $C>T$ transitions than UVA or unirradiated controls of the same genotype (Figure 4A\&C, Suppl. Table 2B; $p<1.02 E-$ 14 and 1.82E-13, respectively). The absolute number of $\mathrm{C}>\mathrm{T}$ mutations was slightly, but not significantly, greater in $T N-U V B$ than $T N-$ NoUV tumors (Figure 4A\&C, Suppl. Table 2B; $p=0.22$ ). However, the relative frequency of $\mathrm{C}>\mathrm{T}$ mutations in both UVB-irradiated models was greater than UVA or unirradiated tumors of the same genotype (Figure 4B, Suppl. Table $2 \mathrm{C}$; $p<3.90 \mathrm{E}-3$ for all comparisons). All groups showed a similar number and percentage of $\mathrm{C}>\mathrm{T}$ alterations at $\mathrm{CpG}$ sites, suggesting that methylated cytosines are not preferentially mutated as a result of UVB irradiation (Figure 4B-C). Indeed, differences in C>T burden and frequency were primarily driven by mutations at non-CpG sites (Suppl. Table 2B-C). The increased frequency of C>T mutations 
179

180

181

182

183

184

185

186

187

188

189

190

191

192

193

194

195

196

197

was accompanied by decreases in the frequency of $\mathrm{T}>\mathrm{C}$ mutations in tumors from both UVBirradiated models (Figure 4B; Suppl. Table 2C). No other mutation types were enriched in a specific genotype or UV irradiation group (Suppl. Table 2B-C).

We looked for evidence of oncogene-dependent mutational enrichments and found that $\mathrm{C}>\mathrm{T}$ transitions were more abundant $(p<9.79 \mathrm{E}-13)$ in TB-UVB tumors than $T N$-UVB tumors (Figure 4C, Suppl. Table 2B). Other mutation types did not differ significantly in number or frequency between the two genotypes (Suppl. Table 2B-C). These data highlight differences in the ability of UVA and UVB to drive melanoma-associated mutations and suggest that an underlying Braf mutation may promote the accumulation of $\mathrm{C}>\mathrm{T}$ transitions.

Studies in cultured fibroblasts and model organisms implicate transcription-coupled repair (TCR) in the rapid repair of UV-induced DNA lesions (Marteijn et al. 2014). Therefore, we investigated whether the SNVs observed in our TN and TB tumors exhibited a strand bias. Mutations in mock- and UVA-irradiated tumors did not exhibit a strand bias, except in the case of T>C transitions, which were enriched on the non-transcribed strand of TB-UVA samples (Figure 4D, Suppl. Table 2D). Tumors from both UVB-treated models showed a bias for C>T mutations on the non-transcribed strand. $C>A, T>A, T>C$ and $T>G$ mutations were also enriched on the nontranscribed strand of $T B-U V B$, but not $T N-U V B$, tumors. This finding suggests a disparity among $T N$ and $T B$ melanomas in the biochemistry, incidence or repair of UV-associated DNA lesions. 
bioRxiv preprint doi: https://doi.org/10.1101/778449; this version posted December 27, 2020. The copyright holder for this preprint (which was A not certified by peer review) is the author/funder. All rights reserved. No reuse allowed without permission.

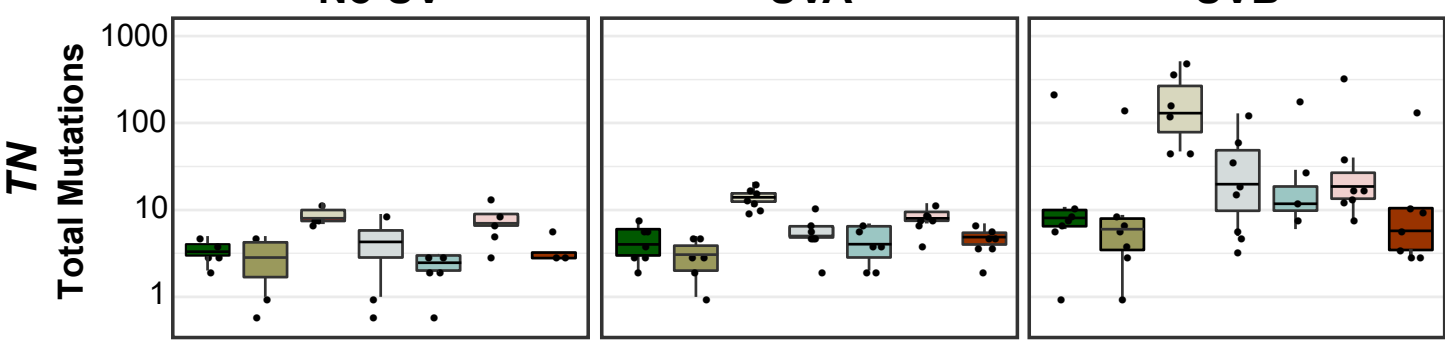

Mutation Type

$\mathrm{C}>\mathrm{A}$

$C>G$

$\mathrm{C}>\mathrm{T}$ other
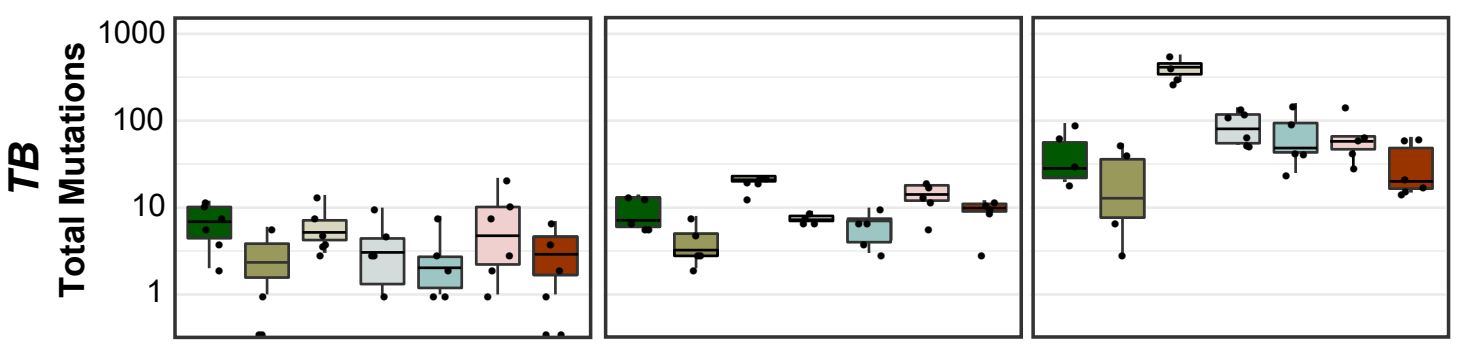

$\mathrm{C}>\mathrm{T}$ at $\mathrm{CpG}$

$\mathrm{T}>\mathrm{A}$

$\mathrm{T}>\mathrm{C}$

$T>G$

B
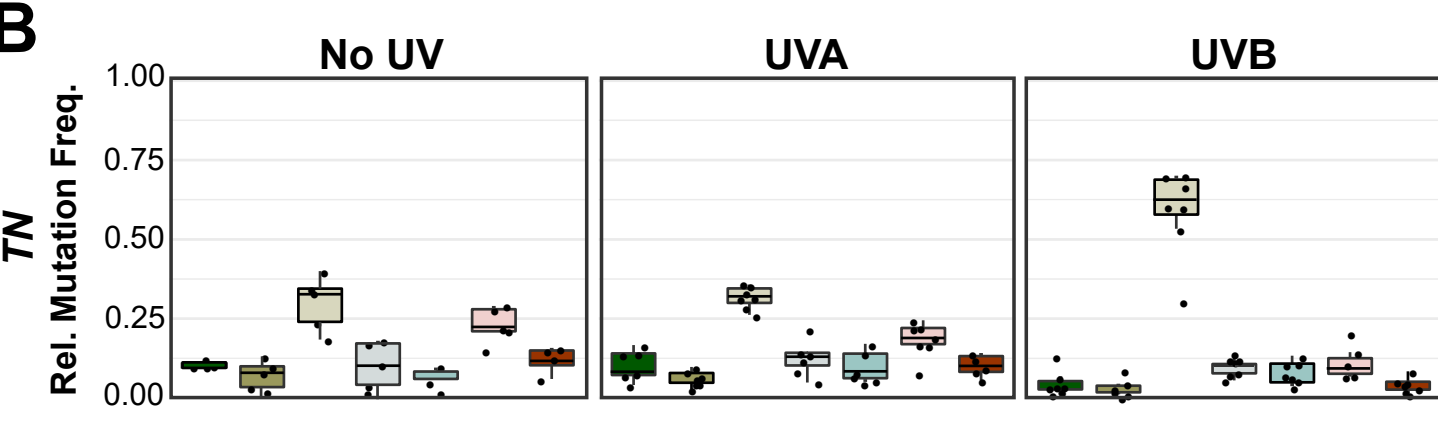

Mutation Type

$C>A$

$C>G$

$\mathrm{C}>\mathrm{T}$ other
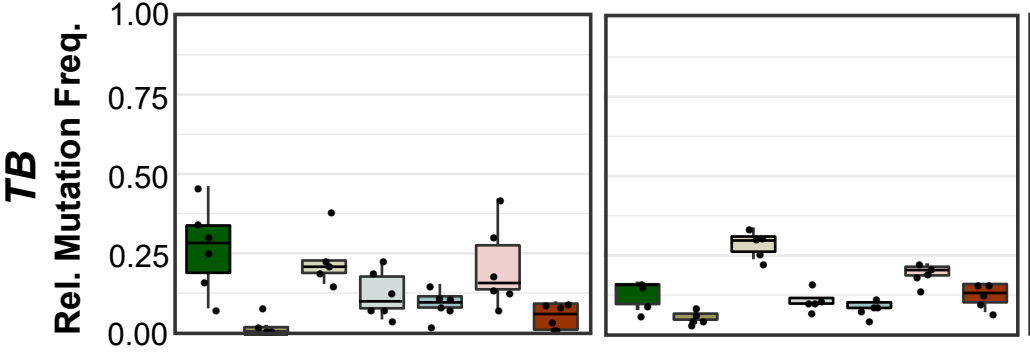

$\mathrm{C}>\mathrm{T}$ at $\mathrm{CpG}$

$T>A$

$\mathrm{T}>\mathrm{C}$

$T>G$

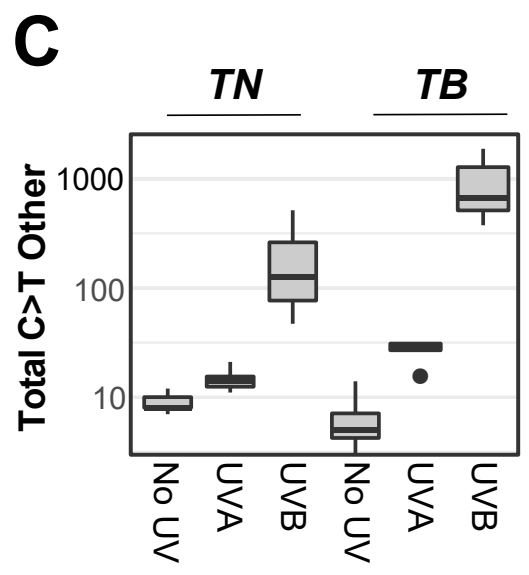

D

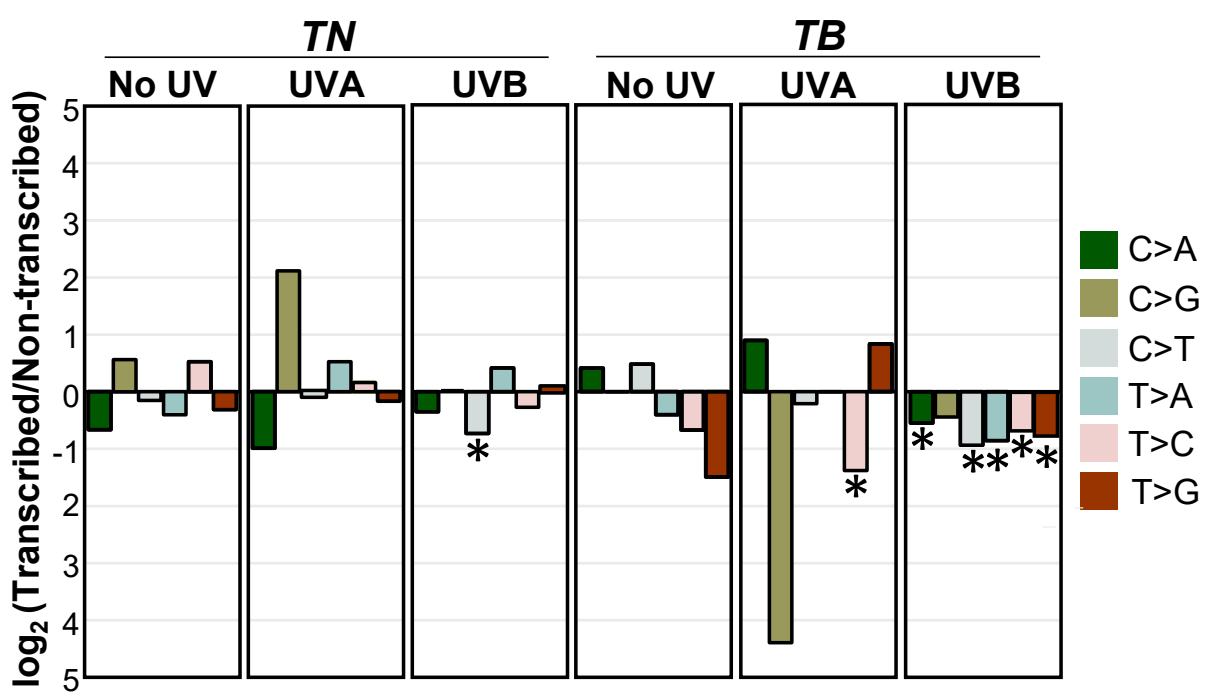

Figure 4. $C>T$ mutations predominate in UVB-induced melanomas. A-B, Absolute mutation burden (A) and frequency (B) of each mutation type in $T N$ and $T B$ tumors arising after mock (No UV), UVA or UVB exposure. See Suppl. Table 2B-C for a complete listing of $p$-values for all comparisons. Statistical significance was evaluated using an ANOVA with Tukey's HSD post-hoc test. C, Boxplot as depicted in (A), restricted to only C>T SNVs at non-CpG locations. D, The strand location of each mutation type was determined using aggregate data from the indicated mouse models and exposures. Plotted are the log-transformed ratios of transcribed versus non-transcribed mutations. Statistical significance of strand bias was assessed using a Poisson test, where an * indicates significant enrichment. Complete p-value listings are found in Suppl. Table 2D. 


\section{Identification of a UVB mutational signature enriched in TB-UVB melanomas}

CPD-associated C>T lesions are considered classical 'UVB signature mutations' and occur

200

201

202

203

204

205

206

207

208

209

210

211

212

213

214

215

216

217

218

219

220

221

222

223

preferentially at dipyrimidine sites (Alexandrov et al. 2013a). C>T transitions in other, noncutaneous cancers lack this specificity (Mitchell et al. 1992). For this reason, we took our sequenced $T N$ and $T B$ melanomas and quantified the burden of each SNV type within every possible trinucleotide context (Suppl. Table 3A). Consistent with these observations, C>T transitions were enriched at TCT and CCT sites in UVB-accelerated TN and TB melanomas (Figure 5A \& B, grey bars). UVB also increased the percentage of C>T mutations at other dipyrimidine sites (CCA, CCC, TCA, TCC and TCG) as compared to No UV control tumors in the TB model. In contrast to UVB-accelerated melanomas, tumors from mock and UVA-irradiated TB and TN mice showed a similar distribution of mutations amongst the sixteen potential trinucleotide sites (Figure 5A \& B). These data are consistent with the pattern of C>T mutations previously observed in a Braf-mutant melanoma mouse model chronically irradiated with UVB (Trucco et al. 2019) and prompted us to further explore whether a mutational signature of UVB exposure might be elucidated from our data.

We used SigProfiler (Alexandrov et al. 2013b) to extract co-occurring mutational patterns, 'mutational signatures', from our complete tumor dataset. This method consistently identified two distinct mutational processes operational in our $T N$ and $T B$ melanomas: Signature 1 and Signature 2 (Figure 6A; Suppl. Table 3B). The profile of Signature 1 contained an abundance of

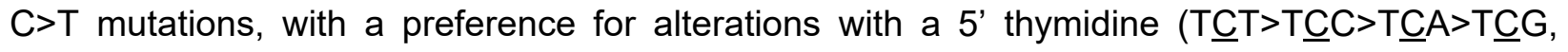
mutated base is underlined). In contrast, the profile of Signature 2 was relatively flat with no specific mutational preference. To ensure that Signatures 1 and 2 were both reproducible and robust, we used two additional algorithms to extract mutational signatures from our complete dataset: SignatureAnalyzer (Kasar et al. 2015) and MutationalPatterns (Blokzijl et al. 2018). Consistent with SigProfiler, SignatureAnalyzer and MutationalPatterns identified two distinct mutational processes in our dataset (Suppl. Figure S4, data not shown). A high degree of 

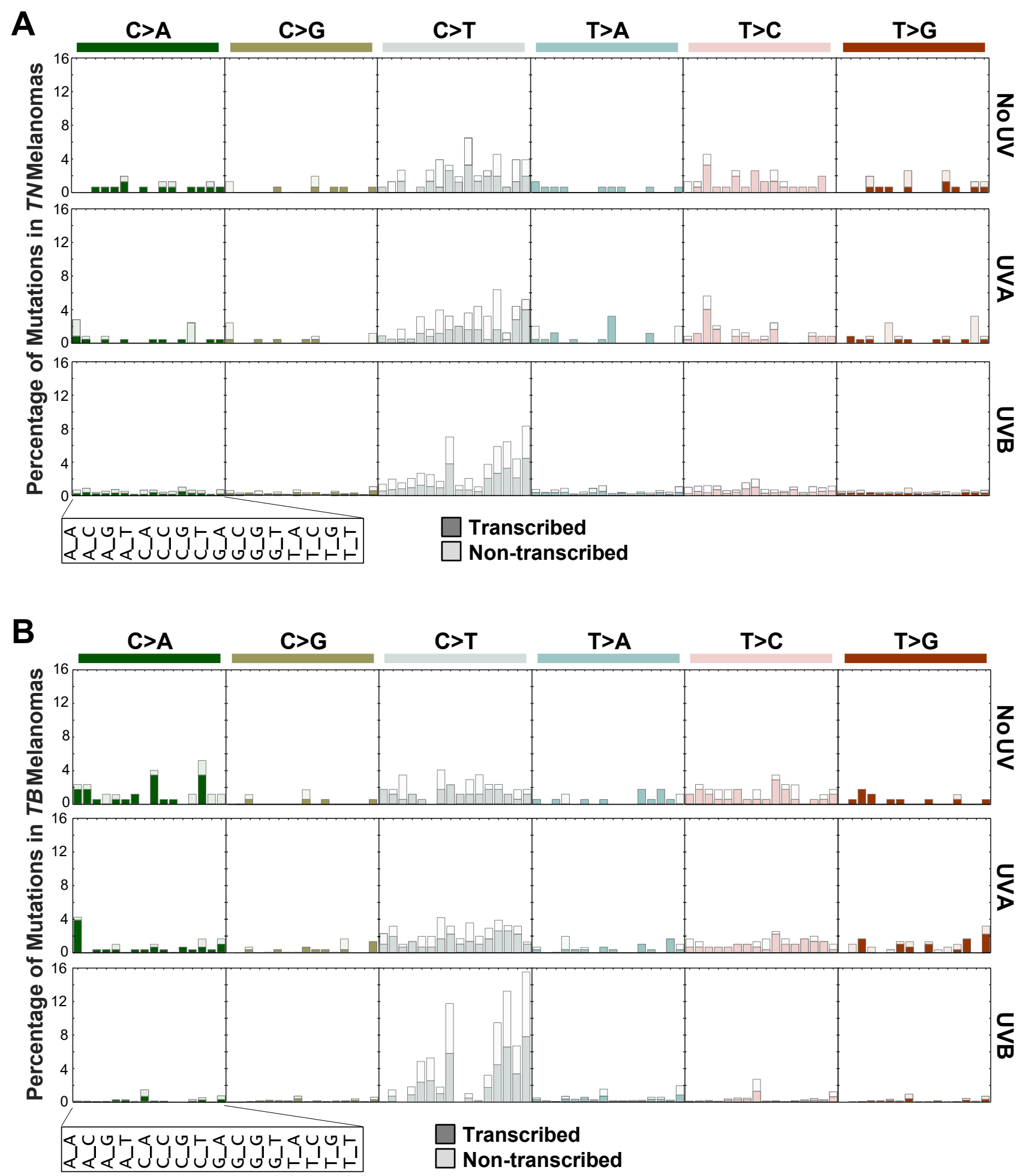

Figure 5. UVB-induced $C>T$ transitions occur within similar trinucleotide contexts in $T N$ and TB melanomas. A-B, Barplots indicating the percentage of each indicated mutation type for a given trinucleotide context in $T N(A)$ and $T B(B)$ tumors. Each subgraph is a mutation type as indicated, where each column within the graph represents a different trinucleotide context surrounding the SNV of interest. Dark shaded bars represent alterations that occur on the transcribed strand while nonshaded bars indicate alterations that occur on the non-transcribed strand. The height of each bar corresponds to the average number of mutations in the indicated experimental group, normalized to the frequency of the relevant trinucleotide sequence in the mouse exome. 
224

225

226

227

228

229

230

231

232

233

234

235

236

237

238

239

240

241

242

243

244

245

246

247 melanoma.

similarity was seen between signatures identified by each algorithm, suggesting that the mutational signatures initially found using SigProfiler were robust (Figure 6B).

We next looked to see if either of the SigProfiler (SP) signatures were enriched in a genotypeor treatment-dependent manner in our mouse melanomas. Signature 2 showed a slight enrichment for pyrimidine transitions, but this enrichement was not specific to any genotype or UV treatment, consistent with the idea that Signature 2 represents background noise or mutagenic process common to all experimental groups (Figure 6C). Signature 1 was enriched in melanomas from UVB-irradiated TN and TB mice over those from No UV- or UVA-treated animals (Figure 6C, Suppl. Table 3C-D). This enrichment was more pronounced in UVB-TB than UVB-TN tumors, indicating a greater effect of UVB on the mutational profile of TB tumors.

The enrichment of Signature 1 in UVB-treated mice suggested that this profile could exhibit features in common with mutational signatures enriched in sun-exposed human tumors. Therefore, we looked at the cosine of similarity between the signatures identified in our murine dataset and the COSMIC mutational signatures. Our Signature 2 was associated with clock-like signatures that correlate with patient chronologic age, including: SBS40 and SBS5 (Figure 6D, Suppl. Table 3E; (Alexandrov et al. 2013a; Alexandrov et al. 2015) . Our Signature 1 closely correlated with SBS7a and 7b (cosine of similiary 0.81 and 0.77 , respectively), which are associated with cancers in sun exposed skin and linked to UV damage (Alexandrov et al. 2013a). Interestingly, there was no significant association between Signature 1 and two other COSMIC signatures, SBS7c and SBS7d, which are enriched in skin cancer and characterized by T>A and T>C SNVs (cosine of similiarity 0.24 and 0.18 respectively) (Alexandrov et al. 2020). These data suggest that mutagenic processes not modeled by our GEMMs may also contribute to human melanomagenesis; however, SBS7a and SBS7b are the predominant signatures found in human 
bioRxiv preprint doi: https://doi.org/10.1101/778449; this version posted December 27, 2020. The copyright holder for this preprint (which was
not certified by peer review) is the author/funder. All rights reserved. No reuse allowed without permission.

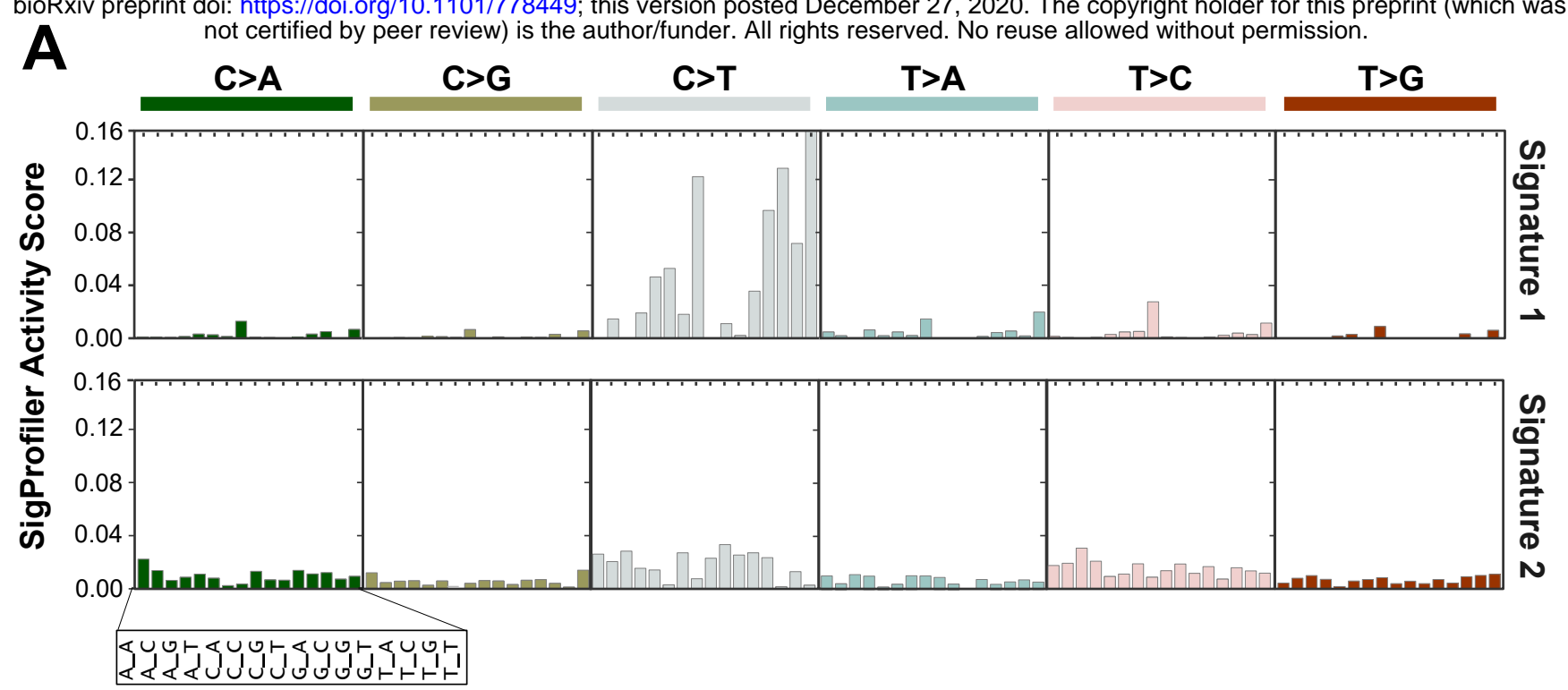

B
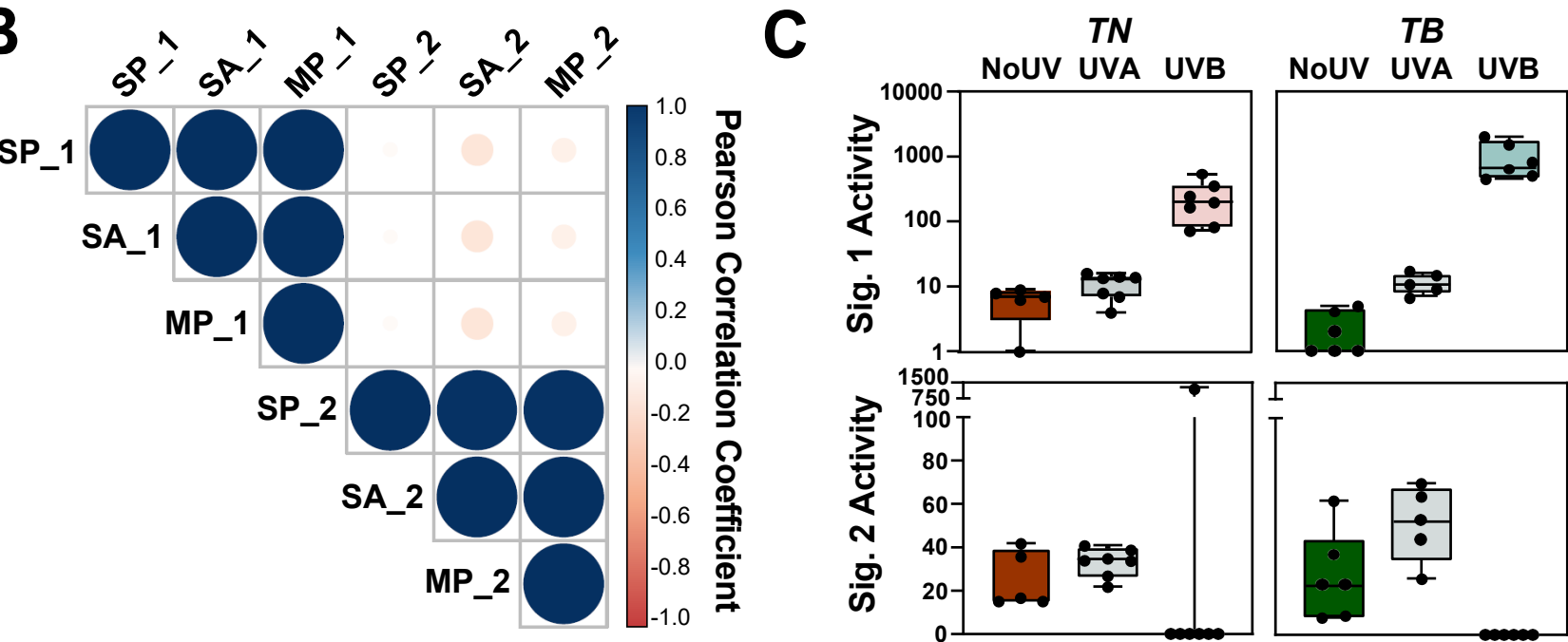

D

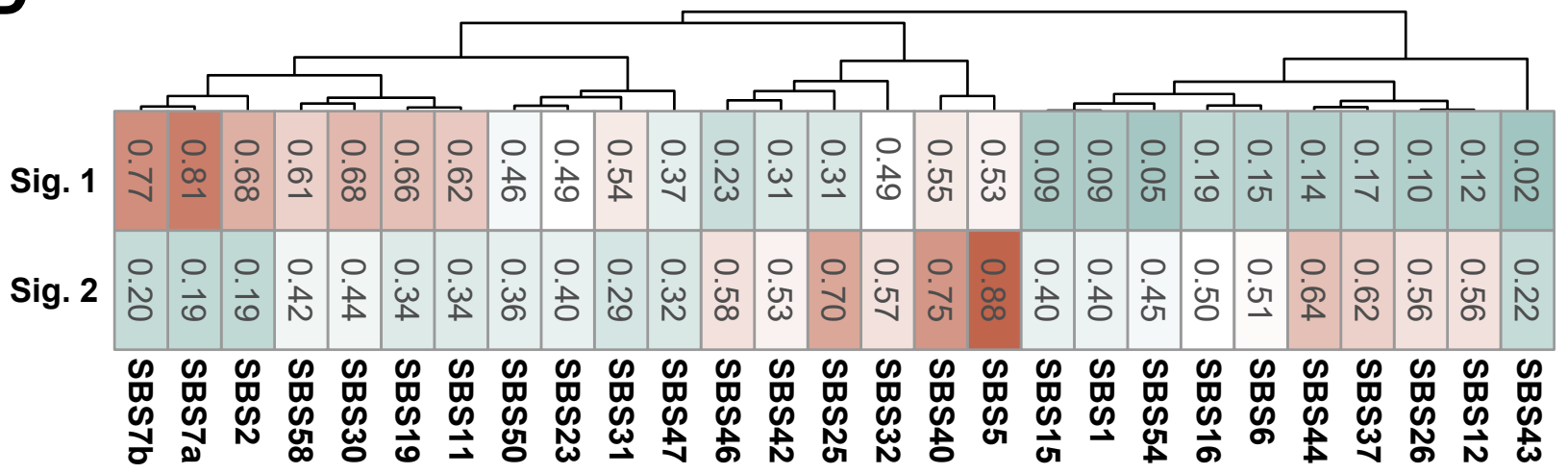

Figure 6. UVB-enriched murine mutational signatures resemble those found in human melanomas. A, SigProfiler was used to identify de novo mutational signatures in sequenced $T N$ and $T B$ melanomas. Two signatures were selected based on the average frobenius reconstruction error and signature stability. The $y$-axis indicates the relative contribution of each trinucleotide mutation type to the discovered mutational signature. Values can be found in Suppl. Table 3B. B, Relationships between signatures derived from SigProfiler (SP_1, SP_2), MutationalPatterns (MP_1, MP_2) and SignatureAnalyzer (SA_1, SA_2) are shown. Color indicates the directionality of each correlation with blue indicating concordance and red indicating discordance. The size of each dot represents the absolute value of the correlation. C, Enrichment of SigProfiler signatures in $T N$ and $T B$ tumors of each exposure type. Significant differences between groups were assessed using an ANOVA with a Fisher's Least Significant Difference post-test. Complete $p$-value listings appear in Suppl. Table 3D and total mutation counts in Suppl. Table 3C. D, Relationship between SigProfiler mutational signatures identified in our dataset (Sig. 1, Sig. 2) and single base substitution (SBS) patterns in the COSMIC database. Suppl. Table 3E contains a complete listing of all $p$-values, which were empirically generated through a cosine similarity permutation test as described in the methods. 


\section{DISCUSSION}

Human melanoma has one of the highest mutational burdens of any tumor type (Alexandrov et al. 2013a). Yet, tumors arising in most melanoma GEMMs, including our unirradiated TN and TB mice, are largely characterized by CNAs rather than SNVs (Hodis et al. 2012; Krauthammer et al. 2012; Zhang et al. 2016; Wang et al. 2017; Zloza et al. 2017). Here we find that a single UVB exposure can resolve this conundrum and effectively recapitulate the high burden of SNVs in human melanoma. Furthermore, the pattern of SNVs in our models is representative of mutational signatures observed in the sun-exposed, human tumors (Figures 3E, 6C-D). The UVB-signature derived from our mice does have a higher number of TCT>TTT variants than SBS7a and SBS7b (Figure 6A; (Alexandrov et al. 2020)) However, this could be attributed to differences in the trinucleotide frequencies found in each species. Variances in the sequences of transcribed mouse and humans genes could also bias which CPD lesions are efficiently targeted by transcription-coupled repair. Of note, human squamous cell carcinomas deficient in global nucleotide excision repair, exhibit a bias for TCT>TTT variants similar to our models (Chang and Shain 2020). Therefore, differences in how human and mouse cells repair UV lesions may explain the increased prevalence of TCT>TTT variants in our UVB signature. The fact that TCT>TTT variants are enriched in other UVB-accelerated GEMMs further supports this hypothesis (Viros et al. 2014; Mukhopadhyay et al. 2016; Trucco et al. 2019).

Our UVB model recapitulates the burden and distribution of SNVs in human melanoma and identifies recurrently mutated genes seen in the human disease. Specifically, we identify clustered Map3k1 and Flna mutations in $T N$ and TB melanomas irrespective of UV-irradiation status (Figure 2). Map3k1 is amplified in a subset of human desmoplastic melanomas (Shain et al. 2015a) and was previously linked to melanoma progression by two, independent transposonmediated mutagenesis screens conducted in the Tyr::CreER(T2) Braf ${ }^{C A}$ model (Ni et al. 2013; Mann et al. 2015). Nevertheless, it remains to be determined how mutations affecting the MAP3K1 RING domain influence tumorigenesis. Similar to MAP3K1, functional defects in Filamin 
A are implicated in the progression of solid tumors (Savoy and Ghosh 2013). In our models, Flna mutations localize primarily to the tenth Ig-like repeat. This domain is responsible for F-actin binding and associated with germline mutations that cause several otopalatodigital spectrum disorders (OSDs) (Moutton et al. 2016). Collectively, these results highlight the potential of forward genetics approaches, like that employed here, to offer insights into the distinct evolutionary trajectories initiated by oncogenic and environmental pressures.

Our results indicate a disparity in the melanomagenic potential of UVA and UVB. Along with prior publications (Noonan et al. 2012; Trucco et al. 2019), these data provide additional evidence that UVA exposures may increase melanoma risk, but not to the same extent as UVB. It is noteworthy that the higher burden of mutations in our UVB tumors did not provide a growth advantage (Figures 1F, 3C). Rather, UVB seems to enhance the ability of genetically predisposed melanocytes to initiate tumor formation. This finding aligns with Blum's interpretation of the kinetics by which UV initiates non-melanoma skin cancers in albino mice (Blum 1969). Specifically, Blum hypothesized that UV-dependent tumor initiation requires a combination of genetic and mitogenic effects. Therefore, in our genetically pre-disposed models, UV-induced growth factors may facilitate initial tumor growth leading to an earlier onset.

What distinguishes this study from past investigations is the direct comparison of UVcarcinogenesis in NRAS- and BRAF-mutant melanoma. While we did not evaluate the response of healthy tissue, this approach did allow us to detect differences in the mutagenicity of a single UVB exposure among melanomas expressing endogenous levels of mutant NRAS or BRAF (Figure 2). It is unlikely that the increased mutational burden of UVB-accelerated TB tumors is solely attributed to the longer latency of these tumors as compared to UVB-initiated $T N$ melanomas (Figure 1C-D). Notably, despite differences in tumor latency, TN and TB melanomas from the UVA and No UV groups exhibit a similar number of SNVs per Mb (Figure $3 \mathrm{C}$ ). Furthermore, differential enrichment of the UVB mutational signature, as well as dipyrimidine substitutions, suggest that UVB carcinogenesis differs between the two models (Figures 3E, 6C). 
Whether this difference explains the increased prevalence of $B R A F$-mutant melanomas on parts of the body intermittently exposed to sunlight requires further investigation.

As mentioned in the introduction, a similar proportion of UV signature lesions $(\mathrm{C}>\mathrm{T}$ and $\mathrm{CC}>\mathrm{TT}$ ) are seen in NRAS- and BRAF-mutant human melanomas, despite the predominance of NRAS-mutant tumors in chronically sun damaged skin (Cancer Genome Atlas 2015). If, as

307 predicted by our data, the presence of a BRAF mutation enhances UVB carcinogenesis, then 308 fewer exposures might be required for melanoma progression. Alternatively, BRAF and NRAS309 mutant cells may exhibit differential thresholds for genotoxic stress-induced apoptosis or senescence.

Understanding how NRAS- and BRAF-mutant melanocytes respond to UVB damage will likely offer insight into the differential SNV burden observed here. Genotype-dependent DNA-damage responses were recently reported in melanoma cell lines (Sauvaigo et al. 2020). However, an earlier report saw no correlation between repair capacity and melanoma genotype (Gaddameedhi et al. 2010). In BRAF-mutant melanomas, loss of p19ARF promotes the epigenetic silencing of XPC, leading to deficiencies in nucleotide excision repair (Luo et al. 2013). Meanwhile,

317 pharmacological inhibition of BRAF has been shown to increase nuclear import of the by-pass 318 polymerase, Pol-K, resulting in increased drug tolerance without clear evidence of enhanced mutagenesis (Temprine et al. 2020). Collectively, these results suggest that melanocyte genotype 320 is likely to modulate the cellular response to UV. However, these studies utilized cell lines or 321 extracts, which fail to model the early stages of melanomagenesis and the potential influence of 322 the tumor microenvironment on repair. We posit that isogenically controlled, in vivo studies will 323 be necessary to understand how distinct oncogenic and mutagenic stresses cooperate to 324 modulate DNA-damage response and cellular fitness. 


\section{METHODS}

\section{Mouse models}

All animal research protocols were approved by The Ohio State University Institutional Animal Care and Use Committee (Protocol \#2012A00000134). Mice were backcrossed $>7$ generations to pigmented, C57BL/6J animals. Inducible knock-in and knockout alleles were activated with 20 mM 4-hydroxytamoxifen on postnatal (p.n.) days one and two as described (Burd et al. 2014). Subjects from each litter were randomly assigned to receive either ambient light (No UV), UVA or UVB on p.n. day three. A single dose of narrow band UVB was delivered to the dorsal side of each animal using a fixed position, 16W, 312nm light source (Spectronics \#EB-280C). Based upon the spectrum and intensity of this light source, we calculated the McKinlay-Diffey erythemal effective energy (EEE) of a $4.5 \mathrm{~kJ} / \mathrm{m}^{2}$ dose, delivered over $\sim 77$ seconds, to be $75 \mathrm{~mJ} / \mathrm{cm}^{2}$ ((Diffey 2002); Suppl. Figure S5A). A dose of $75 \mathrm{~mJ} / \mathrm{cm}^{2}$ EEE UVB is equivalent to 3.9 human minimal erythema doses (MEDs) in an individual with phototype II skin (i.e. someone who tans minimally, but usually burns with red/blond hair and blue/green/hazel eyes) or to approximately 40 minutes of sun exposure when the UV index is Very High [see (Hennessey et al. 2017) for additional information]. UVA was similarly delivered using a $16 \mathrm{~W}$ source containing two BLE-8T365 bulbs

342 (Spectronics). Based upon the spectrum of these bulbs, the calculated McKinlay-Diffey erythemal effective energy (EEE) of a $70 \mathrm{~kJ} / \mathrm{m}^{2}$ dose is $14.2 \mathrm{~mJ} / \mathrm{cm}^{2}$ ((Diffey 2002); Suppl. Figure S5B).

344 The average tanning parlor dose is 4.5 Standard Erythema Doses (SEDs; (Dowdy et al. 2011). 345 One SED is equivalent to $10 \mathrm{~mJ} / \mathrm{cm}^{2}$ EEE-weighted UV light (Diffey 2002). Therefore, an 346 individual receives $>3$ times more UVA in an average tanning session than a mouse in our 70 $347 \mathrm{~kJ} / \mathrm{m}^{2}$ experimental protocol $\left(45 \mathrm{~mJ} / \mathrm{cm}^{2} / 14.2 \mathrm{~mJ} / \mathrm{cm}^{2}=3.17\right)$.

\section{Tumor monitoring, processing and histopathology}

350 Protocols for tumor monitoring, processing and immunoblotting appear in the Supplemental

351 Methods. Tumor morphology was assessed by a certified member of the American College of 
352 Veterinary Pathologists (KMDL) using methods described by Banerjee and Harris (Banerjee and

353 Harris 2000). In each sample the extent of skin and subcutis tumor invasion, tumor pigmentation

354 and the maximum number of mitotic figures were determined from three different fields of view

355 using a 40x objective and 10x ocular lens with a field number of $22 \mathrm{~mm}$.

\section{Whole exome sequencing}

Tumor and germline control DNA was isolated and quality controlled as described in the

Supplemental Methods. Indexed libraries were generated from $200 \mathrm{ng}$ of genomic DNA using the Kapa Hyper Prep and Agilent SureSelectXT Mouse All Exon target enrichment systems. Exome hybridization was conducted using $500 \mathrm{ng}$ of each DNA library and the resulting targetenriched fragments PCR-amplified (11 cycles). Indexed libraries were pooled and subjected to paired-end $150 \mathrm{bp}$ sequencing on an Illumina HiSeq4000. Average target coverage was 75X (range 53 - 107X). An overview of WES mapping and coverage metrics appears in Supplemental

Table 4.

\section{Variant Calling}

Sequences were aligned to mm10 using BWA (version 0.7.15) (Li and Durbin 2009). around indels using GATK version 3.6 (McKenna et al. 2010). Variants were called using

371 VarScan2 (version 2.4) (Koboldt et al. 2012), Mutect2 (Cibulskis et al. 2013) and Strelka2 (Kim et 372 al. 2018). Variants identified by all three callers were filtered to remove existing variations in the

373 Ensembl mouse variation database (Yates et al. 2020) and annotated using Variant Effect 374 Predictor (McLaren et al. 2016). Over 200 calls across samples were visually inspected for depth, 375 alignment and read quality in Integrated Genomics Viewer (IGV; (Robinson et al. 2011)). 376 Dipyrimidine mutations were counted as a single event when calculating total mutational burden. 


\section{Analysis of SNVs and CNAs}

SNV burden (variants/Mb) was calculated as a function of the total capture region. SNVs occurring within a dipyrimidine sites were counted as a single event. Oncoprints of genes mutated in three of more mouse melanomas were made with ComplexHeatmap version 2.0.0 (Gu et al. 2016). To calculate the overlap with human tumors, CNAs were identified using CNVkit (Talevich et al. 2016). Reported CNAs passed a $\log _{2}$ segmentation threshold of 0.2 with support from at least five bins. Genome fraction containing a CNA was determined by computing the footprint of segments surpassing the copy number threshold and dividing this by the total footprint of all segments.

\section{Mutational spectrum analysis}

The total burden and relative contribution of each mutation type to No UV-, UVA- and UVBinduced melanomas was determined using the "mut_type_occurrences" algorithm in the R package for MutationalPatterns (Blokzijl et al. 2018). Differences in the absolute number of Holm's adjustment for multiple comparisons ( $p<0.05$ considered significant).

A MATLAB implementation of SigProfiler (Alexandrov 2020) and an R implementation of 397 SignatureAnalyzer (Kim et al. 2016) were used to identify de novo mutational signatures. Average 398 Frobenius reconstruction error and signature stability were used to select the number of 399 signatures in SigProfiler. The number of signatures selected by SignatureAnalyzer was 400 determined using a Bayesian NMF model described previously (Kim et al. 2016), where two 401 signatures were the most frequent selection from 20 iterations. MutationalPatterns was used to 402 examine strand bias and identify de novo mutational signatures (Blokzijl et al. 2018). The number 403 of signatures was selected using non-negative matrix factorization, and a rank of 2 was chosen 
based on maximization of variance explained and cophenetic score. Comparison of de novo

405 mutational signatures from SigProfiler and those appearing in COSMIC version 3 (Alexandrov et

406 al. 2020) was completed using a cosine of similarity test, for which empirical $p$-values were

407 generated based on 1,000,000 permutations using the "cosinePerm" function from the

408 PharmacoGx package (Smirnov et al. 2016).

409

410

DATA ACCESS

411 All raw sequencing data generated in this study have been submitted to NCBI Sequence Read

412 Archive (SRA) (https://www.ncbi.nlm.nih.gov/sra) under accession \#PRJNA574176. Code and

413 scripts can be found at https://github.com/bowmanr/UV_mouse_melanoma.

\section{ACKNOWLEDGMENTS}

416 This work was supported by the Melanoma Research Alliance (309669 to C.E.B.), Damon Runyon

417 Foundation (38-16 to C.E.B; 22-17 to R.L.B.), Pelotonia (R.C.H., E.R.C.) and The National

418 Institutes of Health (F31CA236418 to B.M.M.; R01 R01CA237213 to C.E.B.; P30CA016058 to

419 The Ohio State University).

420

\section{DISCLOSURE DECLARATION}

422 R.L.L. is on the supervisory board of Qiagen and is a scientific advisor to Imago, Mission Bio,

423 Zentalis, Ajax, Auron, Prelude, C4 Therapeutics and Isoplexis. He receives research support from

424 and consulted for Celgene and Roche and has consulted for Incyte, Janssen, Astellas, Morphosys

425 and Novartis. He has received honoraria from Roche, Lilly and Amgen for invited lectures and

426 from Gilead for grant reviews. 


\section{REFERENCES}

428

429

430

431

432

433

434

435

436

437

438

439

440

441

442

443

444

445

446

447

448

449

450

451

452

453

454

455

456

457

458

459

460

461

462

463

464

465

466

467

468

469

470

471

472

473

Alexandrov LB. 2020. SigProfiler In MATLAB Central File Exchange.

Alexandrov LB, Jones PH, Wedge DC, Sale JE, Campbell PJ, Nik-Zainal S, Stratton MR. 2015. Clock-like mutational processes in human somatic cells. Nature genetics 47: 1402-1407.

Alexandrov LB, Kim J, Haradhvala NJ, Huang MN, Tian Ng AW, Wu Y, Boot A, Covington KR, Gordenin DA, Bergstrom EN et al. 2020. The repertoire of mutational signatures in human cancer. Nature $\mathbf{5 7 8 :}$ 94-101.

Alexandrov LB, Nik-Zainal S, Wedge DC, Aparicio SA, Behjati S, Biankin AV, Bignell GR, Bolli N, Borg A, Borresen-Dale AL et al. 2013a. Signatures of mutational processes in human cancer. Nature 500: 415-421.

Alexandrov LB, Nik-Zainal S, Wedge DC, Campbell PJ, Stratton MR. 2013b. Deciphering signatures of mutational processes operative in human cancer. Cell Rep 3: 246-259.

Banerjee SS, Harris M. 2000. Morphological and immunophenotypic variations in malignant melanoma. Histopathology 36: 387-402.

Bishop JA, Wachsmuth RC, Harland M, Bataille V, Pinney E, Mac KP, Baglietto L, Cuzick J, Bishop DT. 2000. Genotype/phenotype and penetrance studies in melanoma families with germline CDKN2A mutations. The Journal of investigative dermatology 114: 28-33.

Blokzijl F, Janssen R, van Boxtel R, Cuppen E. 2018. MutationalPatterns: comprehensive genome-wide analysis of mutational processes. Genome Med 10: 33.

Blum H. 1969. Quantitative aspects of cancer induction by ultraviolet light: including a revised model In: Urbach F (ed). The Biologic Effects of Ultraviolet Radiation-with Emphasis on the Skin. Pergamon Press: New York.

Bosenberg M, Muthusamy V, Curley DP, Wang Z, Hobbs C, Nelson B, Nogueira C, Horner JW, 2nd, Depinho R, Chin L. 2006. Characterization of melanocyte-specific inducible Cre recombinase transgenic mice. Genesis 44: 262-267.

Burd CE, Liu W, Huynh MV, Waqas MA, Gillahan JE, Clark KS, Fu K, Martin BL, Jeck WR, Souroullas GP et al. 2014. Mutation-specific RAS oncogenicity explains NRAS codon 61 selection in melanoma. Cancer discovery 4: 1418-1429.

Cadet J, Douki T. 2018. Formation of UV-induced DNA damage contributing to skin cancer development. Photochem Photobiol Sci 17: 1816-1841.

Cadet J, Wagner JR. 2013. DNA base damage by reactive oxygen species, oxidizing agents, and UV radiation. Cold Spring Harb Perspect Biol 5.

Cancer Genome Atlas N. 2015. Genomic Classification of Cutaneous Melanoma. Cell 161: 1681-1696.

Chagani S, Wang R, Carpenter EL, Lohr CV, Ganguli-Indra G, Indra AK. 2017. Ablation of epidermal RXRalpha in cooperation with activated CDK4 and oncogenic NRAS generates spontaneous and acute neonatal UVB induced malignant metastatic melanomas. BMC Cancer 17: 736.

Chang D, Shain AH. 2020. The landscape of driver mutations in cutaneous squamous cell carcinoma. bioRxiv doi:10.1101/2020.12.13.422581: 2020.2012.2013.422581.

Cibulskis K, Lawrence MS, Carter SL, Sivachenko A, Jaffe D, Sougnez C, Gabriel S, Meyerson M, Lander ES, Getz G. 2013. Sensitive detection of somatic point mutations in impure and heterogeneous cancer samples. Nat Biotechnol 31: 213-219.

Dankort D, Curley DP, Cartlidge RA, Nelson B, Karnezis AN, Damsky WE, Jr., You MJ, DePinho RA, McMahon M, Bosenberg M. 2009. Braf(V600E) cooperates with Pten loss to induce metastatic melanoma. Nature genetics 41: 544-552.

Dankort D, Filenova E, Collado M, Serrano M, Jones K, McMahon M. 2007. A new mouse model to explore the initiation, progression, and therapy of BRAFV600E-induced lung tumors. Genes \& development 21: 379-384. 
Diffey BL. 2002. Sources and measurement of ultraviolet radiation. Methods 28: 4-13.

Dowdy JC, Czako EA, Stepp ME, Schlitt SC, Bender GR, Khan LU, Shinneman KD, Karos MG, Shepherd JG, Sayre RM. 2011. FDA-sunlamp recommended Maximum Timer Interval And Exposure Schedule: consensus ISO/CIE dose equivalence. Health Phys 101: 227-232.

Gaddameedhi S, Kemp MG, Reardon JT, Shields JM, Smith-Roe SL, Kaufmann WK, Sancar A. 2010. Similar nucleotide excision repair capacity in melanocytes and melanoma cells. Cancer Res 70: 49224930.

Gu Z, Eils R, Schlesner M. 2016. Complex heatmaps reveal patterns and correlations in multidimensional genomic data. Bioinformatics 32: 2847-2849.

Hennessey RC, Holderbaum AM, Bonilla A, Delaney C, Gillahan JE, Tober KL, Oberyszyn TM, Zippin JH, Burd CE. 2017. Ultraviolet radiation accelerates NRas-mutant melanomagenesis: A cooperative effect blocked by sunscreen. Pigment cell \& melanoma research 30: 477-487.

Hodis E, Watson IR, Kryukov GV, Arold ST, Imielinski M, Theurillat JP, Nickerson E, Auclair D, Li L, Place C et al. 2012. A landscape of driver mutations in melanoma. Cell 150: 251-263.

Kasar S, Kim J, Improgo R, Tiao G, Polak P, Haradhvala N, Lawrence MS, Kiezun A, Fernandes SM, Bahl S et al. 2015. Whole-genome sequencing reveals activation-induced cytidine deaminase signatures during indolent chronic lymphocytic leukaemia evolution. Nat Commun 6: 8866.

Khan AQ, Travers JB, Kemp MG. 2018. Roles of UVA radiation and DNA damage responses in melanoma pathogenesis. Environ Mol Mutagen 59: 438-460.

Kim J, Mouw KW, Polak P, Braunstein LZ, Kamburov A, Kwiatkowski DJ, Rosenberg JE, Van Allen EM, D'Andrea A, Getz G. 2016. Somatic ERCC2 mutations are associated with a distinct genomic signature in urothelial tumors. Nature genetics 48: 600-606.

Kim S, Scheffler K, Halpern AL, Bekritsky MA, Noh E, Kallberg M, Chen X, Kim Y, Beyter D, Krusche P et al. 2018. Strelka2: fast and accurate calling of germline and somatic variants. Nat Methods 15: 591594.

Kitts A, Phan L, Ward MH, Holmes JB. 2013. The Database of Short Genetic Variation (dbSNP). In The NCBI Handbook National Center for Biotechnology Information, Bethesda (MD).

Koboldt DC, Zhang Q, Larson DE, Shen D, McLellan MD, Lin L, Miller CA, Mardis ER, Ding L, Wilson RK. 2012. VarScan 2: somatic mutation and copy number alteration discovery in cancer by exome sequencing. Genome research 22: 568-576.

Krauthammer M, Kong Y, Ha BH, Evans P, Bacchiocchi A, McCusker JP, Cheng E, Davis MJ, Goh G, Choi M et al. 2012. Exome sequencing identifies recurrent somatic RAC1 mutations in melanoma. Nature genetics 44: 1006-1014.

Li H, Durbin R. 2009. Fast and accurate short read alignment with Burrows-Wheeler transform. Bioinformatics 25: 1754-1760.

Luo C, Sheng J, Hu MG, Haluska FG, Cui R, Xu Z, Tsichlis PN, Hu GF, Hinds PW. 2013. Loss of ARF sensitizes transgenic BRAFV600E mice to UV-induced melanoma via suppression of XPC. Cancer Res 73: 4337-4348.

Mann MB, Black MA, Jones DJ, Ward JM, Yew CC, Newberg JY, Dupuy AJ, Rust AG, Bosenberg MW, McMahon $M$ et al. 2015. Transposon mutagenesis identifies genetic drivers of Braf(V600E) melanoma. Nature genetics 47: 486-495.

Marteijn JA, Lans H, Vermeulen W, Hoeijmakers JH. 2014. Understanding nucleotide excision repair and its roles in cancer and ageing. Nat Rev Mol Cell Biol 15: 465-481.

McKenna A, Hanna M, Banks E, Sivachenko A, Cibulskis K, Kernytsky A, Garimella K, Altshuler D, Gabriel S, Daly M et al. 2010. The Genome Analysis Toolkit: a MapReduce framework for analyzing nextgeneration DNA sequencing data. Genome research 20: 1297-1303.

McLaren W, Gil L, Hunt SE, Riat HS, Ritchie GR, Thormann A, Flicek P, Cunningham F. 2016. The Ensembl Variant Effect Predictor. Genome Biol 17: 122. 
Mitchell DL, Jen J, Cleaver JE. 1992. Sequence specificity of cyclobutane pyrimidine dimers in DNA treated with solar (ultraviolet B) radiation. Nucleic Acids Res 20: 225-229.

Monahan KB, Rozenberg GI, Krishnamurthy J, Johnson SM, Liu W, Bradford MK, Horner J, Depinho RA, Sharpless NE. 2010. Somatic p16(INK4a) loss accelerates melanomagenesis. Oncogene 29: 58095817.

Moutton S, Fergelot P, Naudion S, Cordier MP, Sole G, Guerineau E, Hubert C, Rooryck C, Vuillaume ML, Houcinat $\mathrm{N}$ et al. 2016. Otopalatodigital spectrum disorders: refinement of the phenotypic and mutational spectrum. J Hum Genet 61: 693-699.

Mukhopadhyay P, Ferguson B, Muller HK, Handoko HY, Walker GJ. 2016. Murine melanomas accelerated by a single UVR exposure carry photoproduct footprints but lack UV signature $\mathrm{C}>\mathrm{T}$ mutations in critical genes. Oncogene 35: 3342-3350.

Nakamura F, Osborn TM, Hartemink CA, Hartwig JH, Stossel TP. 2007. Structural basis of filamin A functions. J Cell Biol 179: 1011-1025.

$\mathrm{Ni}$ TK, Landrette SF, Bjornson RD, Bosenberg MW, Xu T. 2013. Low-copy piggyBac transposon mutagenesis in mice identifies genes driving melanoma. Proc Natl Acad Sci U S A 110: E36403649.

Noonan FP, Zaidi MR, Wolnicka-Glubisz A, Anver MR, Bahn J, Wielgus A, Cadet J, Douki T, Mouret S, Tucker MA et al. 2012. Melanoma induction by ultraviolet $A$ but not ultraviolet $B$ radiation requires melanin pigment. Nat Commun 3: 884 .

Page RC, Clark JG, Misra S. 2011. Structure of filamin A immunoglobulin-like repeat 10 from Homo sapiens. Acta Crystallogr Sect F Struct Biol Cryst Commun 67: 871-876.

Perez-Guijarro E, Day CP, Merlino G, Zaidi MR. 2017. Genetically engineered mouse models of melanoma. Cancer 123: 2089-2103.

Pfeifer GP, You YH, Besaratinia A. 2005. Mutations induced by ultraviolet light. Mutat Res 571: 19-31.

Rad R, Cadinanos J, Rad L, Varela I, Strong A, KriegI L, Constantino-Casas F, Eser S, Hieber M, Seidler B et al. 2013. A genetic progression model of $B r a f(V 600 E)$-induced intestinal tumorigenesis reveals targets for therapeutic intervention. Cancer cell 24: 15-29.

Robinson JT, Thorvaldsdottir H, Winckler W, Guttman M, Lander ES, Getz G, Mesirov JP. 2011. Integrative genomics viewer. Nat Biotechnol 29: 24-26.

Roh MR, Eliades P, Gupta S, Tsao H. 2015. Genetics of melanocytic nevi. Pigment cell \& melanoma research 28: 661-672.

Sauvaigo S, Benkhiat M, Braisaz F, Girard J, Libert S, Mouret S, de Fraipont F, Aspord C, Bouquet F, Leccia $M-T$. 2020. DNA repair-based classification of melanoma cell lines reveals an effect of mutations in BRAF and NRAS driver genes on DNA repair capacity. bioRxiv doi:10.1101/2020.04.29.067900: 2020.2004.2029.067900.

Savoy RM, Ghosh PM. 2013. The dual role of filamin A in cancer: can't live with (too much of) it, can't live without it. Endocr Relat Cancer 20: R341-356.

Setlow RB. 1974. The wavelengths in sunlight effective in producing skin cancer: a theoretical analysis. Proc Natl Acad Sci U S A 71: 3363-3366.

Shain AH, Garrido M, Botton T, Talevich E, Yeh I, Sanborn JZ, Chung J, Wang NJ, Kakavand H, Mann GJ et al. 2015a. Exome sequencing of desmoplastic melanoma identifies recurrent NFKBIE promoter mutations and diverse activating mutations in the MAPK pathway. Nature genetics 47: 11941199.

Shain AH, Joseph NM, Yu R, Benhamida J, Liu S, Prow T, Ruben B, North J, Pincus L, Yeh I et al. 2018. Genomic and Transcriptomic Analysis Reveals Incremental Disruption of Key Signaling Pathways during Melanoma Evolution. Cancer cell 34: 45-55 e44. 
Shain AH, Yeh I, Kovalyshyn I, Sriharan A, Talevich E, Gagnon A, Dummer R, North J, Pincus L, Ruben B et al. 2015b. The Genetic Evolution of Melanoma from Precursor Lesions. N Engl J Med 373: 19261936.

Smirnov P, Safikhani Z, El-Hachem N, Wang D, She A, Olsen C, Freeman M, Selby H, Gendoo DM, Grossmann P et al. 2016. PharmacoGx: an R package for analysis of large pharmacogenomic datasets. Bioinformatics 32: 1244-1246.

Suphamungmee W, Nakamura F, Hartwig JH, Lehman W. 2012. Electron microscopy and 3D reconstruction reveals filamin Ig domain binding to F-actin. J Mol Biol 424: 248-256.

Sutherland JC, Griffin KP. 1981. Absorption spectrum of DNA for wavelengths greater than $300 \mathrm{~nm}$. Radiat Res 86: 399-409.

Talevich E, Shain AH, Botton T, Bastian BC. 2016. CNVkit: Genome-Wide Copy Number Detection and Visualization from Targeted DNA Sequencing. PLoS computational biology 12: e1004873.

Temprine K, Campbell NR, Huang R, Langdon EM, Simon-Vermot T, Mehta K, Clapp A, Chipman M, White RM. 2020. Regulation of the error-prone DNA polymerase Polkappa by oncogenic signaling and its contribution to drug resistance. Sci Signal 13.

Trucco LD, Mundra PA, Garcia-Martinez P, Hogan K, Baenke F, Dhomen N, Pavet V, Marais R. 2020. Map3k1 Loss Cooperates with Braf(V600E) to Drive Melanomagenesis. The Journal of investigative dermatology doi:10.1016/j.jid.2020.05.085.

Trucco LD, Mundra PA, Hogan K, Garcia-Martinez P, Viros A, Mandal AK, Macagno N, Gaudy-Marqueste C, Allan D, Baenke F et al. 2019. Ultraviolet radiation-induced DNA damage is prognostic for outcome in melanoma. Nat Med 25: 221-224.

Viros A, Sanchez-Laorden B, Pedersen M, Furney SJ, Rae J, Hogan K, Ejiama S, Girotti MR, Cook M, Dhomen $\mathrm{N}$ et al. 2014. Ultraviolet radiation accelerates BRAF-driven melanomagenesis by targeting TP53. Nature 511: 478-482.

Wang J, Perry CJ, Meeth K, Thakral D, Damsky W, Micevic G, Kaech S, Blenman K, Bosenberg M. 2017. UV-induced somatic mutations elicit a functional T cell response in the YUMMER1.7 mouse melanoma model. Pigment cell \& melanoma research 30: 428-435.

Yates AD, Achuthan P, Akanni W, Allen J, Allen J, Alvarez-Jarreta J, Amode MR, Armean IM, Azov AG, Bennett R et al. 2020. Ensembl 2020. Nucleic Acids Res 48: D682-D688.

Zhang T, Dutton-Regester K, Brown KM, Hayward NK. 2016. The genomic landscape of cutaneous melanoma. Pigment cell \& melanoma research 29: 266-283.

Zloza A, Karolina Palucka A, Coussens LM, Gotwals PJ, Headley MB, Jaffee EM, Lund AW, Sharpe AH, Sznol M, Wainwright DA et al. 2017. Workshop on challenges, insights, and future directions for mouse and humanized models in cancer immunology and immunotherapy: a report from the associated programs of the 2016 annual meeting for the Society for Immunotherapy of cancer. $J$ Immunother Cancer 5: 77. 


\section{Supplemental Materials}

UVB mutagenesis differs in NRAS- and BRAF-mutant mouse models of melanoma

Bowman and Hennessey, et al.

Contents

Pages

Supplemental Figures S1-5.

Supplemental Methods. 
A

TN

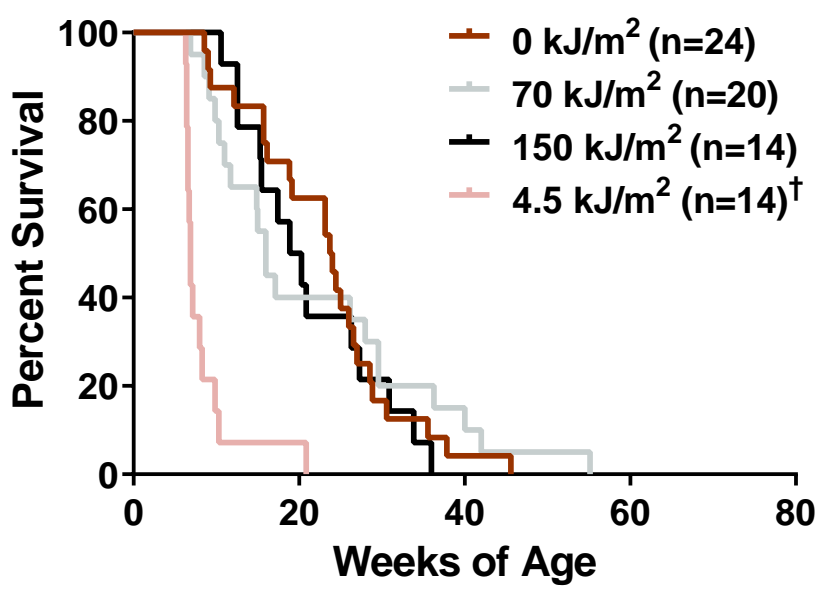

B

TB

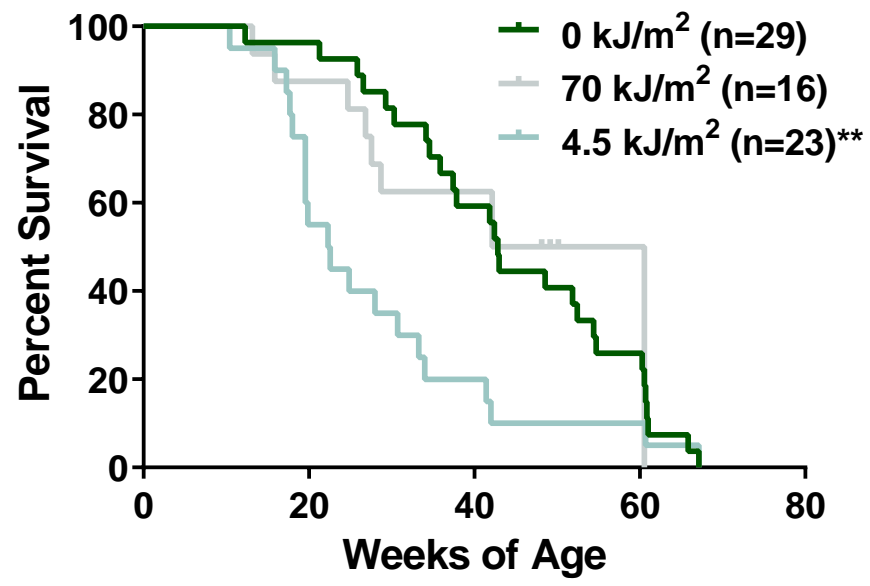

Figure S1. Overall survival of TN and TB mice treated with No UV, UVA or UVB. A\&B, Kaplan-Meier curves depicting the overall survival rates of $T N$ and $T B$ mice treated on postnatal day three with a single dose of ambient light $\left(0 \mathrm{~kJ} / \mathrm{m}^{2}\right)$, UVA or UVB. Mice were euthanized after meeting pre-determined exclusion criteria due to tumor burden or malaise. Statistical significance was determined by comparing values from control $\left(0 \mathrm{~kJ} / \mathrm{m}^{2}\right)$ and UV-treated animals of the same genotype using Gehan-Breslow-Wilcoxon tests. $\dagger=$ $p<0.0001,{ }^{* *}=p<0.01$ 
A

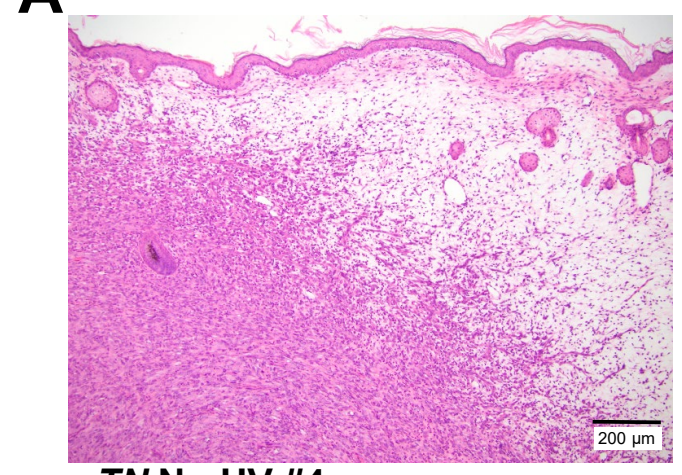

C TN No UV \#4

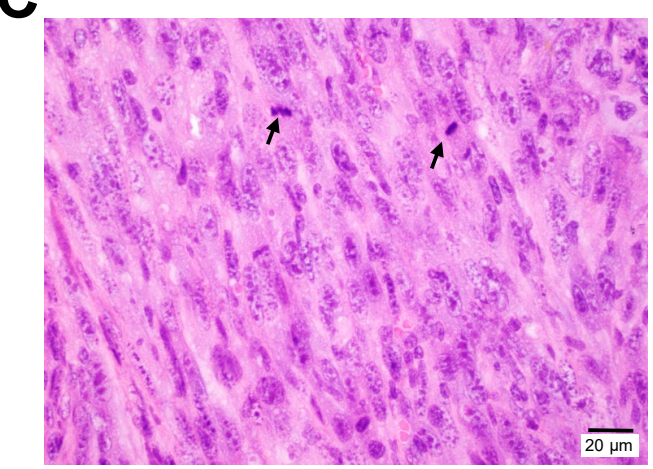

\section{E}

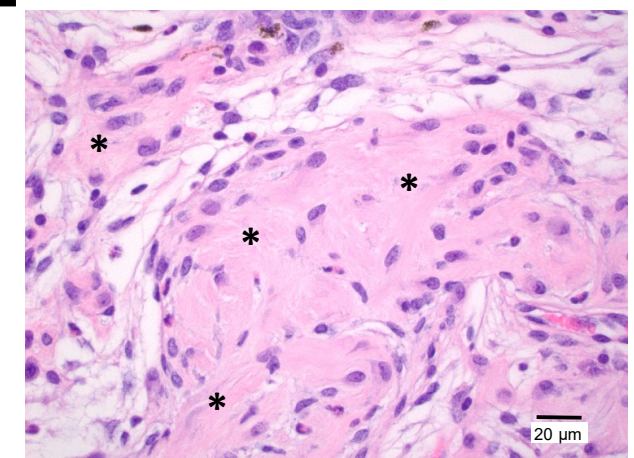

TB No UV \#4
B

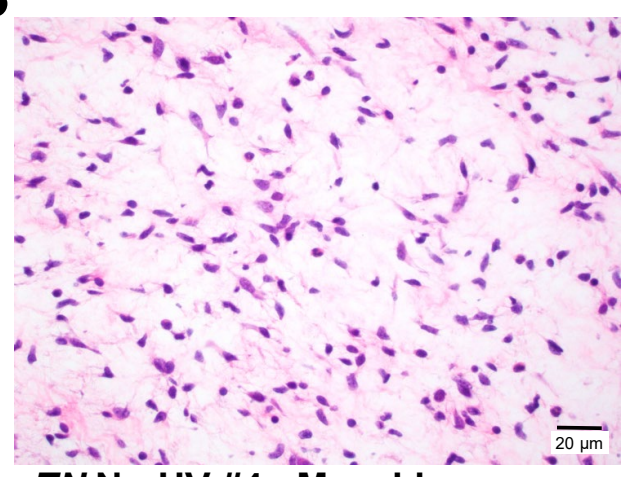

TN No UV \#4 - Myxoid

D

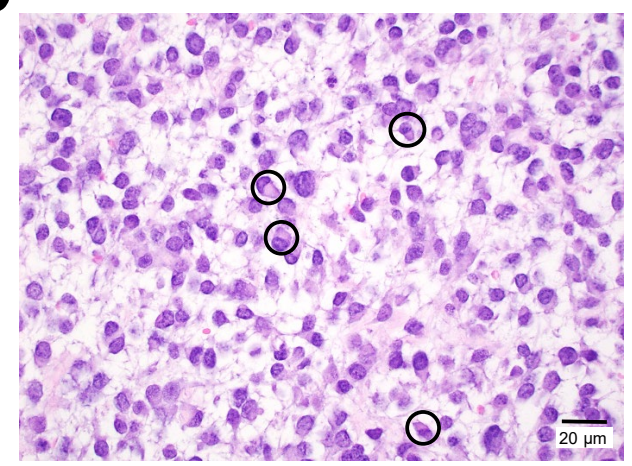

$\mathbf{F}$

TN UVB \#8

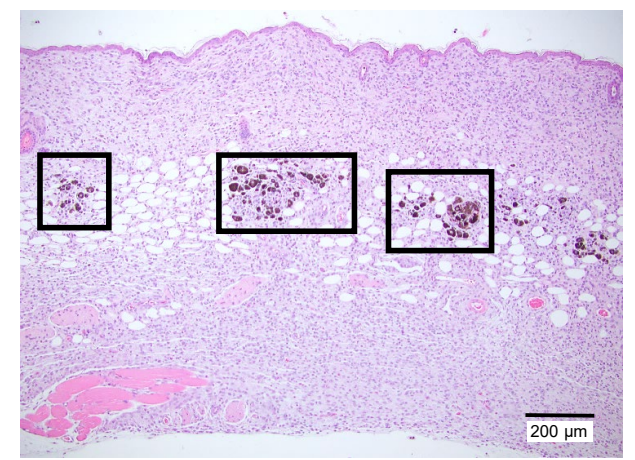

TB UVA \#16

Figure S2. Representative photomicrographs of cutaneous tumors from TN and TB mice. A, $T N$ and TB tumors, regardless of UV exposure history, contain variable proportions of spindle (left, cellular) and myxoid (right, spaces) morphologies. Bar $=200 \mu \mathrm{m}$. B\&C, Higher magnification images of the myxoid ('B', bar $=20 \mu \mathrm{m}$ ) and spindle ('C', bar $=20 \mu \mathrm{m}$ ) morphologies depicted in ' $A$ '. Arrows = mitotic figures. D, Representative image of the neoplastic cells with plasmacytoid features seen in the majority of $T N$ tumors evaluated. (circles; bar $=20 \mu \mathrm{m}$ ). E, Half of the tumors in UVA-treated $T N$ mice exhibited a fibroblastic phenotype with abundant collagen (indicated by *; bar $=20 \mu \mathrm{m}$ ). $\mathbf{F}$, Tumor samples from TB mice contained areas of pigmentation, typically appearing as clusters of melanophages at the dermal-hypodermal interface (boxes, bar $=200 \mu \mathrm{m}$ ). All images are of H\&E stained tissues. 
A
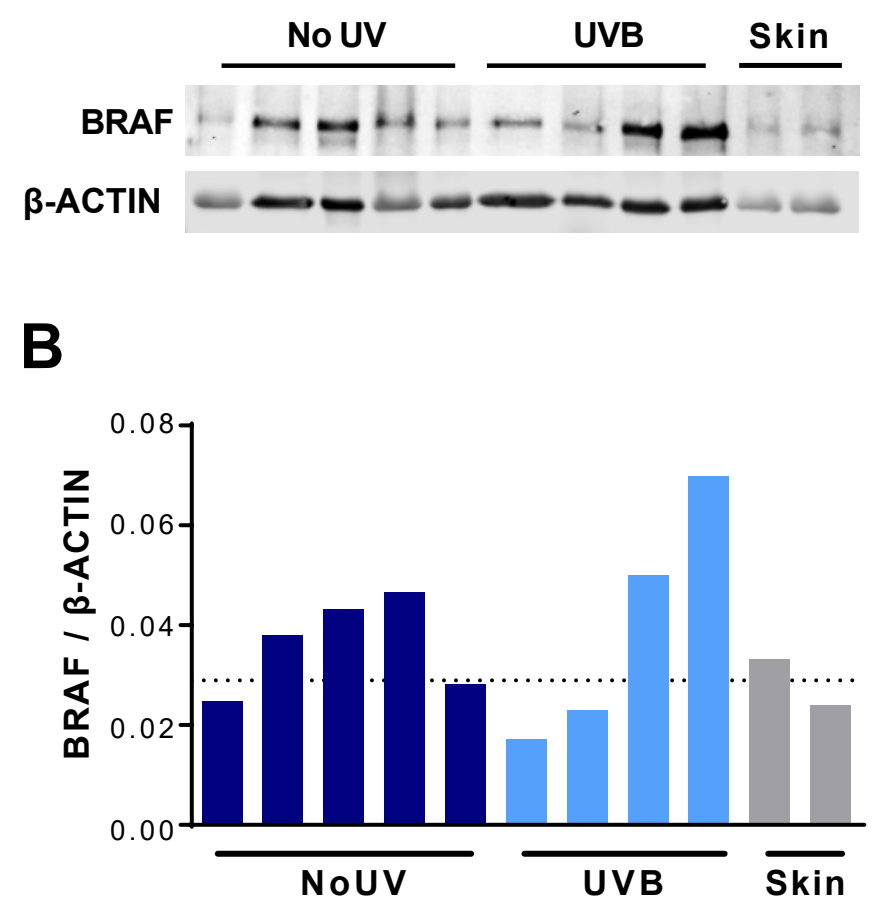

Figure S3. Characterization of BRAF levels in TB tumors. A, Immunoblot of BRAF and $\beta$ ACTIN expression in representative TB tumors and whole skin. Each row contains lysate from a single tumor or skin lysate. B, Quantification of the immunoblot shown in ' $A$ '. The dotted line represents the average BRAF expression level in whole skin. 
A

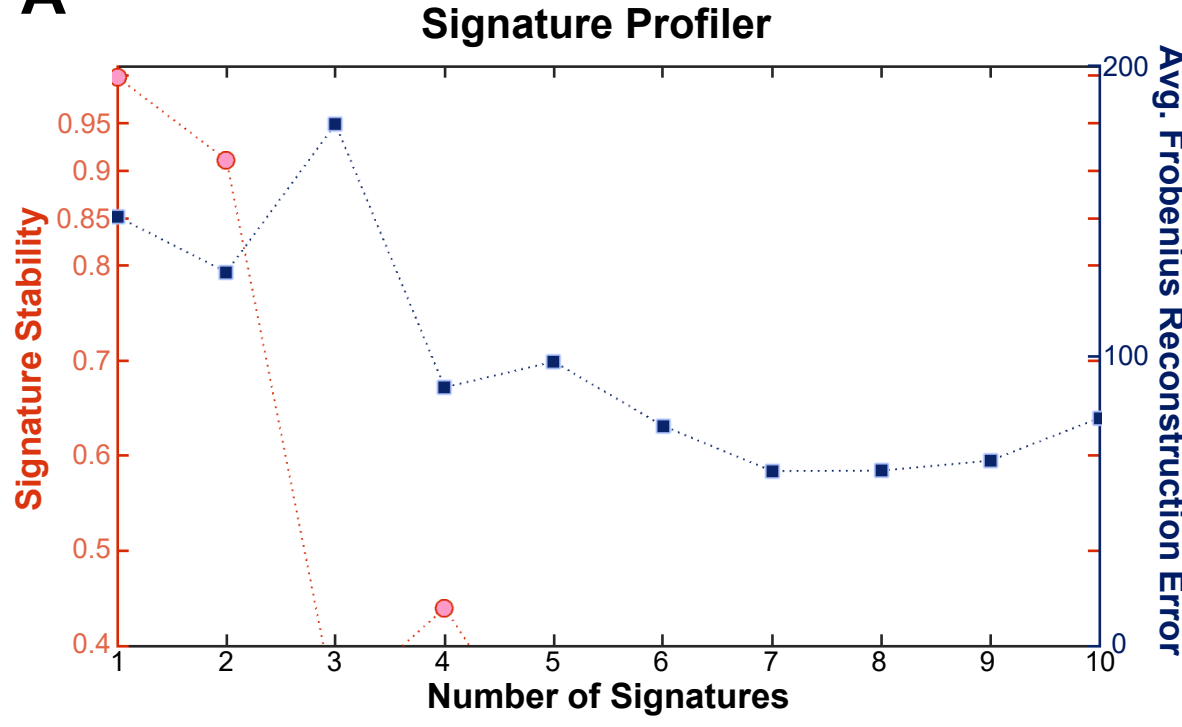

B

\section{Signature Analyzer}

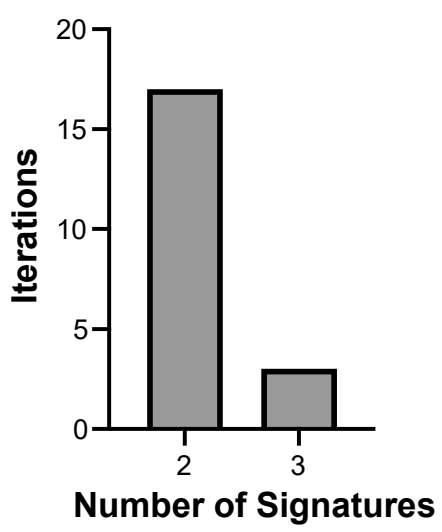

C

NMF rank survey
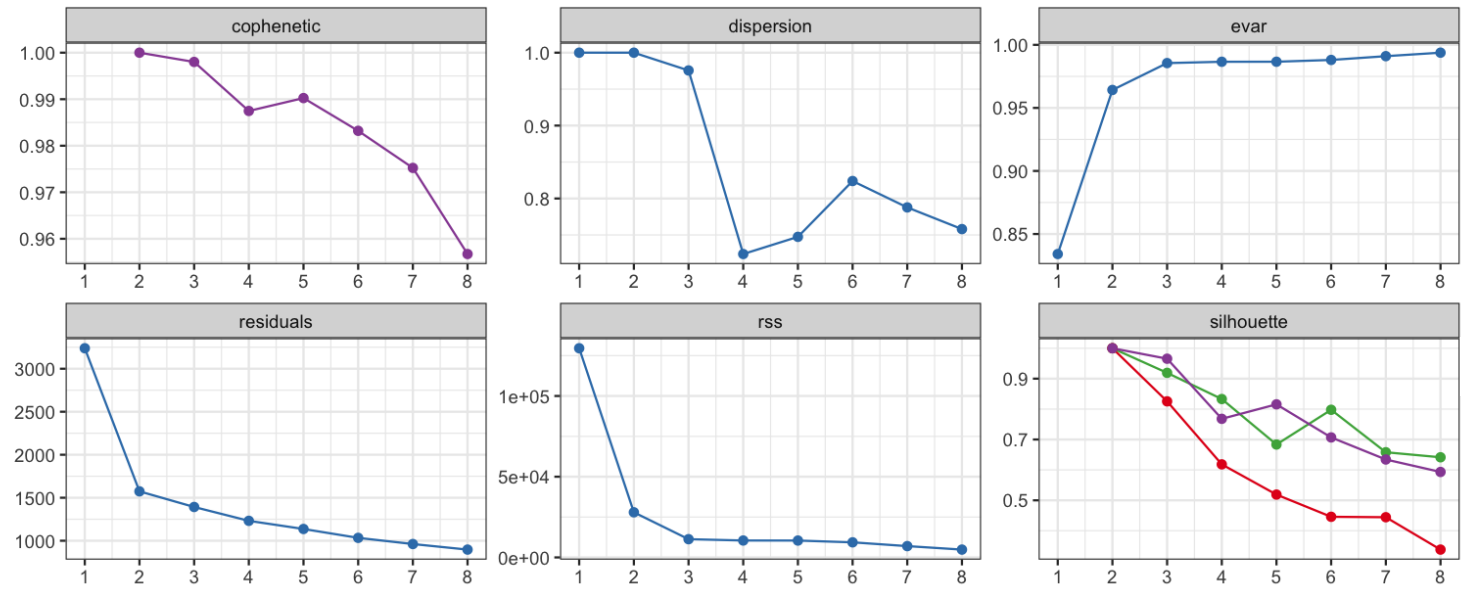

Measure Type

$\rightarrow$ Basis

$\rightarrow$ Best fit

$\rightarrow$ Coefficients

$\rightarrow$ Consensus

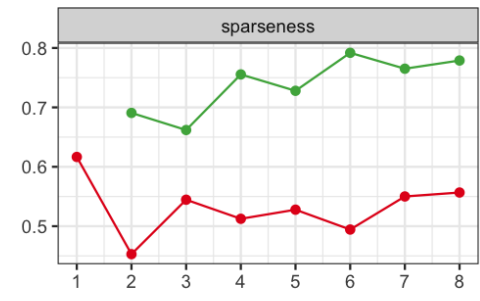

Factorization rank

Figure S4. Signature selection metrics. A, The mean cosine distance representative of signature stability is plotted on the left $y$ axis, and the average frobenius reconstruction error is plotted on the right $y$ axis for each number of signatures tested by SignatureProfiler indicated on the $x$ axis. B, The frequency of signatures identified by Signature Analyzer is shown, with 2 signatures being identified most frequently across the 20 iterations. See methods for details. C, Quality metrics produced for NMF estimate as used in MutationalPatterns. Two signatures were selected as the appropriate estimate based on the cophenetic score as described in the methods. 

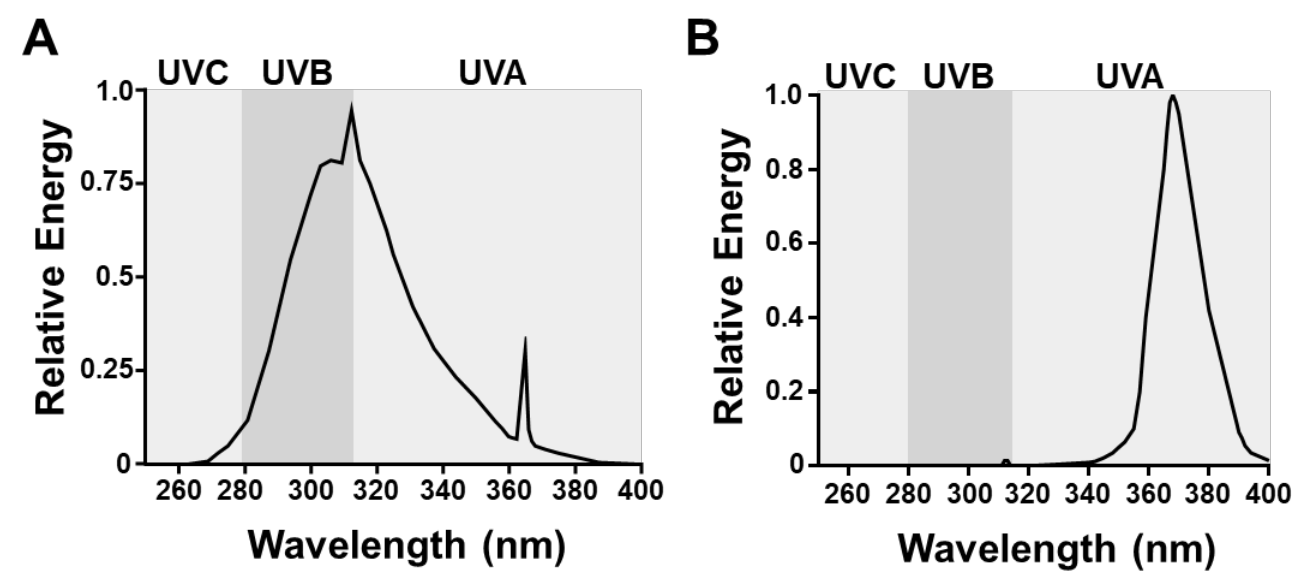

Figure S5. UV light sources. A, Shown is the relative amount of energy produced across the UV spectrum by our narrow band UVB, Spectronics EB-280C light source. B, Shown is the relative amount of energy produced across the UV spectrum by our narrow band UVAI (340-400 $\mathrm{nm})$, BLE-8T365 light source. 
SUPPLEMENTAL METHODS

\section{Tumor monitoring and processing}

62 Mice were randomly numbered following treatment and blindly monitored three times a week for

63 tumor formation. Established melanomas were measured by calipers at least three times per

64 week and tumor size (width $\times$ length $(\mathrm{mm}))$ recorded until protocol exclusion criteria were met.

65 Representative tumors were harvested from each cohort, fixed in $10 \%$ neutral buffered formalin,

66 routinely processed and embedded in paraffin wax. Sections $(4 \mu \mathrm{m})$ were stained with

67 hematoxylin and eosin (H\&E), and evaluated by a veterinary pathologist, certified by the American

68 College of Veterinary Pathologists (KMDL), using an Olympus BX45 light microscope with

69 attached DP25 digital camera (B\&B Microscopes Limited, Pittsburgh, PA).

70

\section{Tumor immunoblotting}

72 Flash-frozen tumors were homogenized in PBS with Halt phosphatase inhibitor (Thermo

73 Scientific) and protease inhibitor (Sigma) using the Precellys Evolution Homogenizer with Cryolys

74 (Bertin Instruments). The preset elastic setting was used for homogenizing. Tumor homogenates

75 were centrifuged to remove PBS and resuspended in RIPA buffer with phosphatase and protease

76 inhibitors. Lysates were sonicated $2 \times 10$ seconds using a Branson digital sonifier at $10 \%$

77 amplitude. Samples were centrifuged at $15,000 \mathrm{rpm}$ for $5 \mathrm{~min}$ at $4^{\circ} \mathrm{C}$ and supernatants collected

78 and quantified by Bradford assay (BioRad). Samples (35 $\mu$ g total) were blotted for BRAF (Santa

79 Cruz sc-5284; $1: 500$ ) and $\beta$-Actin (Cell Signaling \#3700; 1:1000) and imaged using a LI-COR

80 Odyssey CLx system. Bands were quantified using Image Studio Version 5.2 software (LI-COR

81 Biosciences).

82

\section{Sample preparation for whole exome sequencing}

84 Tumor DNA was isolated from flash frozen tissue using the Quick-DNA Miniprep Plus Kit (Zymo 85 Research). Tissues were placed in $2 \mathrm{~mL}$ tubes containing $190 \mu \mathrm{L}$ of diluted Zymo Solid Tissue 
86 Buffer and $3.0 \mathrm{~mm}$ zirconium beads (Sigma Aldrich Cat\# Z763902). Samples were then subjected

87 to homogenization using the Precellys Evolution Homogenizer (Bertin Instruments) using the

88 preset elastic setting: speed: 6,800 RPM, cycle: 4 X $30 \mathrm{sec}$, pause: $45 \mathrm{sec}$. Homogenized

89 samples were then incubated in $20 \mathrm{mg} / \mathrm{mL}$ of Proteinase $\mathrm{K}$ overnight at $55^{\circ} \mathrm{C}$ before continuing

90 with the Solid Tissues protocol as described for the Quick-DNA Miniprep Plus Kit. Control DNA

91 was generated from toe clips or splenic tissue derived from ten representative $T N$ and ten

92 representative $T B$ animals. These controls were then combined at an equal ratio and

93 concentrated using the Genomic DNA Clean \& Concentrator-10 kit (Zymo Research). The

94 integrity and concentration of resulting genomic DNA was confirmed on an Agilent TapeStation. 